University of Nebraska - Lincoln

DigitalCommons@University of Nebraska - Lincoln

$7-1-2004$

\title{
Annual Report to the Nation on the Status of Cancer, 1975-2001, with a Special Feature Regarding Survival
}

\author{
Ahmedin Jemal \\ American Cancer Society, Atlanta, ajemal@cancer.org \\ Limin X. Clegg \\ National Cancer Institute, lin_clegg@va.gov \\ Elizabeth Ward \\ American Cancer Society \\ Lynn A. G. Ries \\ National Cancer Institute, Bethesda, MD \\ Xiao-Cheng Wu \\ North American Association of Central Cancer Registries, xwu@lsuhsc.edu \\ See next page for additional authors \\ Follow this and additional works at: https://digitalcommons.unl.edu/publichealthresources \\ Part of the Public Health Commons
}

Jemal, Ahmedin; Clegg, Limin X.; Ward, Elizabeth; Ries, Lynn A. G.; Wu, Xiao-Cheng; Jamison, Patricia; Wingo, Phyllis A.; Howe, Holly L.; Anderson, Robert N.; and Edwards, Brenda K., "Annual Report to the Nation on the Status of Cancer, 1975-2001, with a Special Feature Regarding Survival" (2004). Public Health Resources. 256.

https://digitalcommons.unl.edu/publichealthresources/256

This Article is brought to you for free and open access by the Public Health Resources at DigitalCommons@University of Nebraska - Lincoln. It has been accepted for inclusion in Public Health Resources by an authorized administrator of DigitalCommons@University of Nebraska - Lincoln. 
Authors

Ahmedin Jemal, Limin X. Clegg, Elizabeth Ward, Lynn A. G. Ries, Xiao-Cheng Wu, Patricia Jamison, Phyllis A. Wingo, Holly L. Howe, Robert N. Anderson, and Brenda K. Edwards 


\section{O M M E N T A R Y}

The authors thank Alicia Samuels and Dama Laurie of the American Cancer Society and Danielle Harkins and Martin Krapcho of Information Management Services, Inc., for assisting with article preparations.

Address for reprints: Ahmedin Jemal, D.V.M., Ph.D., Epidemiology and Surveillance Research, American Cancer Society, 1599 Clifton Road, AtIanta, GA 30329; Fax: (404) 327-6450; E-mail: ajemal@cancer.org

Received March 22, 2004; accepted March 23, 2004.

${ }^{*}$ This article is a US Government work and, as such, is in the public domain in the United States of America.

\section{Annual Report to the Nation on the Status of Cancer, 1975-2001, with a Special Feature Regarding Survival}

\author{
Ahmedin Jemal, D.v.M., Ph.D. ${ }^{1}$ \\ Limin X. Clegg, Ph.D. ${ }^{2}$ \\ Elizabeth Ward, Ph.o. ${ }^{1}$ \\ Lynn A. G. Ries, M.s. ${ }^{2}$ \\ Xiaocheng Wu, M.D., M.P.H., C.T.R. ${ }^{3,4}$ \\ Patricia M. Jamison, M.P.H. ${ }^{5}$ \\ Phyllis A. Wingo, Ph.D. ${ }^{5}$ \\ Holly L. Howe, Ph.D. ${ }^{4}$ \\ Robert N. Anderson, Ph.D. ${ }^{6}$ \\ Brenda K. Edwards, Ph.D. ${ }^{2}$ \\ ${ }^{1}$ Epidemiology and Surveillance Research Department, American Cancer Society, Atlanta, Georgia. \\ ${ }^{2}$ Division of Cancer Control and Population Sciences, National Cancer Institute, Bethesda, Maryland. \\ ${ }^{3}$ School of Public Health, Louisiana State University Health Science Center, Louisiana State University, \\ New Orleans, Louisiana. \\ ${ }^{4}$ North American Association of Central Cancer Registries, Springfield, Illinois. \\ ${ }^{5}$ Division of Cancer Prevention and Control, National Center for Chronic Disease Prevention and \\ Health Promotion, Centers for Disease Control and Prevention, Atlanta, Georgia. \\ ${ }^{6}$ Division of Vital Statistics, National Center for Health Statistics, Centers for Disease Control and \\ Prevention, Hyattsville, Maryland.
}

BACKGROUND. The American Cancer Society (ACS), the Centers for Disease Control and Prevention (CDC), the National Cancer Institute (NCI), and the North American Association of Central Cancer Registries (NAACCR) collaborate annually to provide updated information regarding cancer occurrence and trends in the U.S. This year's report features a special section on cancer survival.

METHODS. Information concerning cancer cases was obtained from the NCI, CDC, and NAACCR and information concerning recorded cancer deaths was obtained from the CDC. The authors evaluated trends in age-adjusted cancer incidence and death rates by regression models and described and compared survival rates over time and across racial/ethnic populations.

RESULTS. Incidence rates for all cancers combined decreased from 1991 through 2001, but stabilized from 1995 through 2001 when adjusted for delay in reporting. The incidence rates for female lung cancer decreased (although not statistically significant for delay adjusted) and mortality leveled off for the first time after increasing for many decades. Colorectal cancer incidence rates also decreased. Death rates decreased for all cancers combined (1.1\% per year since 1993) and for many of the top 15 cancers occurring in men and women. The 5 -year relative survival rates improved for all cancers combined and for most, but 
not all, cancers over 2 diagnostic periods (1975-1979 and 1995-2000). However, cancer-specific survival rates were lower and the risk of dying from cancer, once diagnosed, was higher in most minority populations compared with the white population. The relative risk of death from all cancers combined in each racial and ethnic population compared with non-Hispanic white men and women ranged from 1.16 in Hispanic white men to 1.69 in American Indian/Alaska Native men, with the exception of Asian/Pacific Islander women, whose risk of 1.01 was similar to that of non-Hispanic white women.

CONCLUSIONS. The continued measurable declines for overall cancer death rates and for many of the top 15 cancers, along with improved survival rates, reflect progress in the prevention, early detection, and treatment of cancer. However, racial and ethnic disparities in survival and the risk of death from cancer, and geographic variation in stage distributions suggest that not all segments of the U.S. population have benefited equally from such advances. Cancer 2004;101:3-27. Published 2004 by the American Cancer Society.*

KEYWORDS: cancer; incidence; mortality; survival; Surveillance, Epidemiology, and End Results (SEER); National Program of Cancer Registries (NPCR); North American Association of Central Cancer Registries (NAACCR); vital statistics; U.S.

$T^{1}$ he American Cancer Society (ACS), the Centers for Disease Control and Prevention (CDC), the National Cancer Institute (NCI), and the North American Association of Central Cancer Registries (NAACCR) collaborate each year to produce an annual report to the nation regarding the current status of cancer in the U.S. The initial report in 1998 documented the first sustained decline in cancer death rates since national record-keeping was instituted in the 1930s. ${ }^{1}$ Subsequent reports generally confirmed this finding and provided updates. ${ }^{2-6}$ Last year's report focused on the use of surveillance data for cancer control. The current report provides incidence and mortality data for all cancers and the top 15 cancers among all races combined and in each major racial/ethnic population (whites, blacks, Asians/Pacific Islanders [API], American Indians/Alaska Natives [AI/AN], and Hispanics/ Latinos). It also features a special section concerning cancer survival over time and by race/ethnicity (nonHispanic whites, Hispanic whites, blacks, API, and $\mathrm{AI} / \mathrm{AN})$.

\section{MATERIALS AND METHODS Cancer Cases and Deaths}

Information regarding newly diagnosed cancer cases in the U.S. is based on data collected by cancer registries participating in the NCI's Surveillance, Epidemiology, and End Results (SEER) Program or the CDC's National Program of Cancer Registries (NPCR). All registries are members of the NAACCR. ${ }^{7}$ With the exception of bladder cancer, the data regarding incidence refer to invasive but not in situ cancers. Beginning with incident cases in 2001, all information concerning primary cancer site and histology was coded ac- cording to the third edition of the International Classification of Diseases for Oncology (ICD-O) ${ }^{8}$ and categorized according to SEER site groups. ${ }^{9}$ To ensure as much comparability as possible between the second and third revisions of the ICD-O, the following inclusions/exclusions were made: borderline tumors of the ovary, refractory anemias, and other myelodysplastic syndromes were excluded, whereas pilocytic astrocytomas were included. The site/histology categories presented for childhood survival are based on site first and then histology to be more comparable to the adult cancers; therefore, this listing is not equivalent to the International Classification of Childhood Cancer. ${ }^{10}$ We defined childhood as age $<20$ years. When applicable, cancer cases were staged as either localized, regional, distant, or unknown according to SEER Summary Stage $1977 .{ }^{11,12}$ Cases identified by death certificate or autopsy only were excluded from staging and survival.

Cancer deaths in the U.S., reported to state vital statistics offices and consolidated into a database by the CDC, through the National Vital Statistics System, ${ }^{13}$ were coded according to the version of the International Classification of Diseases (ICD) in use in the U.S. at the time of death. ${ }^{14-16}$ Beginning with 1999 mortality data, the tenth revision of the ICD (ICD-10) was used to code the cause of death. Under ICD-10 rules, cancer was slightly more likely to be selected as the underlying cause of death than under previous ICD rules. ${ }^{17}$ Sites were grouped by SEER to allow for maximum comparability between versions of ICD codes. $^{9}$

For the long-term trend analyses of the rates for all cancers and the 15 most common cancers among 
all races/ethnicity combined, we used SEER incidence data from the 9 original registries (covering approximately $10 \%$ of the U.S. population $)^{18}$ and U.S. mortality data for 1975-2001. For the short-term trend analyses of the incidence and death rates for the 15 most common cancers in each major racial and ethnic population (whites, blacks, API, AI/AN, and Hispanics/ Latinos), we used incidence data from 12 SEER cancer registries (covering approximately $14 \%$ of the U.S. population, including $12 \%$ of whites, $12 \%$ of blacks, $36 \%$ of API, $21 \%$ of AI/AN, and $22 \%$ of Hispanics/ Latinos) and $100 \%$ of the U.S. population for cancer mortality data for 1992-2001. We examined incidence data for 23 cancers and mortality data for 22 cancers to accommodate the top 15 cancers for incidence rates and for death rates in each racial and ethnic population. Information regarding cancer incidence and death is collected on groups within the API and Hispanic/Latino populations but rates cannot be calculated because of the lack of intercensal county population estimates from the U.S. Census Bureau.

Changes in survival rates over time were examined for the top 15 cancers in males and females and for cancers common in children and adolescents (ages birth-19 years) using cancer cases diagnosed during 1975-1979 and 1995-2000 in the 9 SEER areas. For describing and comparing cancer survival rates among racial and ethnic populations, cancer cases diagnosed between 1992-2000 in the 12 SEER areas were used. For assessing geographic variations in stage distribution for cancers of the colon/rectum, female breast, cervix uteri, and prostate, we used incidence data reported to the NAACCR from 29 SEER and NPCR registries for 1996-2000 that met NAACCR criteria for high quality incidence data as of December 2003 , covering $43 \%$ of the U.S. population.

\section{Cancer Incidence and Death Rates}

Rates were age-adjusted to the 2000 U.S. standard million population and expressed per 100,000 populations by use of population estimates from the U.S. Census Bureau. Estimates of rates, standard errors, and $95 \%$ confidence intervals were generated using SEER*Stat software. ${ }^{18}$

\section{Statistical Analysis}

The long-term trends (1975-2001) in cancer incidence and death rates among all races combined were described by joinpoint regression analysis, which involves fitting a series of joined straight lines on a log scale to the trends in the age-adjusted rates. ${ }^{19}$ The resultant trends of varying time periods were described by annual percent change (i.e., the slope of the line segment). We present the incidence trends based on both the observed data and data with reporting adjustment. Reporting adjustment refers to the adjustment for the late arrival of new cancer cases to cancer registries after the standard reporting period for a diagnosis year as well as to changes and updates of data items such as demography and tumor characteristics for previously reported cases. This adjustment, which will be referred to as delay adjustment hereafter, is important because the most recent diagnosis year will have the largest underreporting of cases, with smaller amounts of underreporting for earlier diagnosis years. We use statistical models to adjust the current cancer count to account for anticipated future improvements to the data based on observed patterns in SEER registries. ${ }^{20}$ Delay adjustment may affect the interpretation of current trends, especially for cancers frequently diagnosed in nonhospital settings such as melanoma, breast cancer, and prostate cancer. However, descriptions of long-term trends in the text are based on the observed data, except when specifically noted. For the short-term trend analyses in each racial and ethnic population, annual percent changes for a fixed time period (1992-2001) were estimated by fitting a linear regression line to the natural logarithms of the rates using calendar year as the independent variable. ${ }^{3}$ In describing trends, the terms "increase" or "decrease" were used when the slope (coefficient) of the trend was statistically significant (two-sided $P$ $<0.05)$ and the terms "stable" or "level" were used otherwise.

Changes in the 5-year relative survival rates for the top 15 cancers and childhood cancers were examined by a life table method for cases diagnosed between 1975-1979 and 1995-2000 and followed for vital status through December 31, 2001. We compared survival rates between these two periods by calculating absolute (1995-2000 rate minus 1975-1979 rate) and proportional (absolute change divided by the 1975-1979 rate) changes. Survival rates by race/ethnicity for the 23 cancers were described using cancer-specific survival rates instead of relative survival rates because reliable expected life tables are not available for the Hispanic white, AI/AN, and API populations to generate valid relative survival estimates. The Kaplan-Meier estimator $^{21}$ was used to estimate the 5-year cancerspecific survival. Both relative and cause-specific survival rates provide an estimate of the likelihood of surviving 5 years if the index cancer was the only cause of death, although the estimation methods differ as described elsewhere. ${ }^{22}$ Differences in cancer survival among racial and ethnic populations (with non-Hispanic whites as the reference group) were assessed by computing relative risks (RRs) (i.e., hazard ratios) of cancer death, after controlling for age (all sites, brain 
cancer, myeloma, and leukemia) or controlling for both age and tumor stage at the time of diagnosis (remaining sites) using Cox regression models, with a maximum of 5 years of follow-up. ${ }^{23}$ Stratified Cox models were used to avoid the assumption of proportional hazards for age and tumor stage. SAS statistical software was used in the analysis, with two-sided $P$ values $<0.05$ considered to indicate statistical significance. ${ }^{24}$ Note that the duration of survival was measured from date of diagnosis and that cases identified by death certificate or autopsy only were excluded from survival analyses because the survival duration was unknown or not applicable.

Additional data concerning cancer incidence and mortality are available from the following URL addresses: www.cancer.org (ACS); www.cdc.gov/cancer/ npcr/index.htm and www.cdc.gov/nchs/about/major/ dvs/mortdata.htm (CDC); and www.naaccr.org/CINAP/ index.htm (NAACCR).

\section{RESULTS \\ Update on the Long-Term Incidence Trends for All Cancers Combined and the Top 15 Cancer Sites for All Races}

Overall cancer incidence rates for all racial and ethnic populations combined declined by $0.5 \%$ per year between 1991-2001 but stabilized between 1995-2001 when adjusted for delay in reporting (Table 1). Incidence rates for all cancers combined stabilized from 1995-2001 both in men and in women, but these rates increased in women by $0.3 \%$ per year since 1987 when adjusted for delay in reporting. For males, cancer incidence rates increased during the last time segment for prostate cancer, melanoma of the skin (melanoma), kidney and renal pelvis (kidney) cancer, and cancer of the esophagus and remained the same for urinary bladder (bladder) cancer, non-Hodgkin lymphoma (NHL), liver and intrahepatic bile duct (liver) cancer, and brain and other nervous system (brain) cancers. Incidence declined for the remaining top 15 cancer sites, which include lung and bronchus (lung), colon/rectum, oral cavity and pharynx (oral cavity), leukemia, stomach, pancreas, and larynx. When rates were adjusted for delayed reporting, the directions of trend changed, with a significant increase noted for bladder cancer and a stabilized trend for leukemia and pancreatic cancer reported. The incidence rates for prostate cancer have had large fluctuations in trend. Rates increased $2.6 \%$ per year between 1975-1988 with a more dramatic increase of $16.4 \%$ per year between 1988-1992. Between 1992-1995, rates decreased by $11.4 \%$ per year, followed by a much smaller increase of $1.4 \%$ per year from 1995 through 2001.

For females, increases in incidence among the top
15 cancers were limited to breast cancer, melanoma, thyroid cancer, bladder cancer, and kidney cancer. Female breast cancer increased $0.4 \%$ per year between 1987-2001, which is a slower rate of increase compared with that of the previous time period (19801987 ) of $3.7 \%$ per year. Trends in the remaining sites were declining or stable, except the NHL trend, which increased by $1.1 \%$ per year when rates were adjusted for delayed reporting. The increase in female lung cancer incidence slowed from $4.6 \%$ per year between 1975-1988 to 1.3\% from 1988-1998, and most important, recently declined (1998-2001), although stabilizing rather than declining when rates were adjusted for reporting delay. Female colorectal cancer rates declined rapidly between 1985-1995, and then had a short period of stabilization followed by a recent (1998-2001) decline of $2.8 \%$ per year.

\section{Update on the Long-Term Mortality Trends for All Cancers Combined and the Top 15 Cancer Sites for All Races}

Overall cancer death rates for all racial and ethnic populations combined decreased by $1.1 \%$ per year from 1993-2001, with the decline found to be more pronounced in men (1.5\% per year from 1993-2001) than in women $(0.8 \%$ per year from $1992-2001)$ (Table 2). Mortality trends for the top 15 cancers differed slightly between men and women. Death rates decreased for 11 of the top 15 cancers in men and for 8 of the top 15 cancers in women. Rates increased for esophageal cancer $(0.5 \%$ per year between $1994-2001)$ and liver cancer (1.4\% per year between 1995-2001) in males, but for none of the top 15 cancers in females. Death rates leveled off for kidney cancer and melanoma in men and for 7 of the top 15 cancers in women. It is interesting to note that female lung cancer death rates between 1995-2001 leveled off for the first time after continuously increasing for many decades. Age-adjusted female lung cancer death rates (deaths per 100,000 females) were virtually unchanged in the last 5 years (40.3 in 1995 and 40.9 in 2001), in contrast with the greater than 2 -fold increase reported from 17.6 in 1975 to 39.3 in 1994.

\section{Incidence and Mortality by Race and Ethnicity (Whites, Blacks, API, Al/AN, and Hispanics/Latinos) between 1992-2001}

The 15 most frequently occurring cancers by sex for incidence and mortality between 1992-2001 were ranked for all races combined and for each racial and ethnic population separately (Tables 3 and 4). Among men, cancers of the prostate, lung, and colon/rectum were the three most common incident cancers in rank order for every racial and ethnic population, except His- 
TABLE 1

SEER Incidence Rate Trends with Joinpoint ${ }^{\mathrm{a}}$ Analyses for 1975 through 2001 for the Top 15 Cancers, ${ }^{\text {b }}$ All Races

\begin{tabular}{|c|c|c|c|c|c|c|c|c|}
\hline & \multicolumn{8}{|c|}{ Joinpoint analyses $(1975-2001)^{c}$} \\
\hline & \multicolumn{2}{|c|}{ Trend 1} & \multicolumn{2}{|c|}{ Trend 2} & \multicolumn{2}{|c|}{ Trend 3} & \multicolumn{2}{|c|}{ Trend 4} \\
\hline & Years & $\mathrm{APC}^{\mathrm{d}}$ & Years & $\mathrm{APC}^{\mathrm{d}}$ & Years & $\mathrm{APC}^{\mathrm{d}}$ & Years & $A P C^{d}$ \\
\hline \multicolumn{9}{|l|}{ All sites ${ }^{\mathrm{e}}$} \\
\hline Both sexes & 1975-1983 & $0.9^{\mathrm{f}}$ & 1983-1991 & $1.8^{\mathrm{f}}$ & 1991-2001 & $-0.5^{\mathrm{f}}$ & & \\
\hline (Delay-adjusted) & 1975-1989 & $1.2^{\mathrm{f}}$ & 1989-1992 & 2.8 & 1992-1995 & -2.1 & 1995-2001 & 0.4 \\
\hline Male & $1975-1989$ & $1.3^{\mathrm{f}}$ & 1989-1992 & $5.1^{\mathrm{f}}$ & $1992-1995$ & $-4.6^{\mathrm{f}}$ & $1995-2001$ & -0.2 \\
\hline (Delay-adjusted) & 1975-1989 & $1.3^{\mathrm{f}}$ & 1989-1992 & $5.2^{\mathrm{f}}$ & 1992-1995 & $-4.7^{\mathrm{f}}$ & 1995-2001 & 0.2 \\
\hline Female & $1975-1979$ & -0.2 & 1979-1987 & $1.5^{\mathrm{f}}$ & 1987-1999 & $0.3^{\mathrm{f}}$ & $1999-2001$ & -1.8 \\
\hline (Delay-adjusted) & $1975-1979$ & -0.2 & 1979-1987 & $1.5^{\mathrm{f}}$ & $1987-2001$ & $0.3^{\mathrm{f}}$ & & \\
\hline \multicolumn{9}{|l|}{ Top 15 for males } \\
\hline Prostate & 1975-1988 & $2.6^{\mathrm{f}}$ & 1988-1992 & $16.4^{\mathrm{f}}$ & 1992-1995 & $-11.4^{\mathrm{f}}$ & 1995-2001 & $1.4^{\mathrm{f}}$ \\
\hline (Delay-adjusted) & $1975-1988$ & $2.6^{\mathrm{f}}$ & 1988-1992 & $16.5^{\mathrm{f}}$ & 1992-1995 & $-11.5^{\mathrm{f}}$ & $1995-2001$ & $2.0^{\mathrm{f}}$ \\
\hline Lung and bronchus & $1975-1982$ & $1.5^{\mathrm{f}}$ & 1982-1992 & $-0.5^{\mathrm{f}}$ & $1992-2001$ & $-2.2^{\mathrm{f}}$ & & \\
\hline (Delay-adjusted) & $1975-1982$ & $1.4^{\mathrm{f}}$ & $1982-1991$ & -0.4 & $1991-2001$ & $-1.9^{\mathrm{f}}$ & & \\
\hline Colon and rectum & $1975-1986$ & $1.1^{\mathrm{f}}$ & 1986-1995 & $-2.1^{\mathrm{f}}$ & $1995-1998$ & 1.0 & 1998-2001 & $-3.5^{\mathrm{f}}$ \\
\hline (Delay-adjusted) & $1975-1986$ & $1.1^{\mathrm{f}}$ & 1986-1995 & $-2.1^{\mathrm{f}}$ & $1995-1998$ & 1.1 & $1998-2001$ & $-2.9^{\mathrm{f}}$ \\
\hline Urinary bladder & $1975-1987$ & $0.9^{\mathrm{f}}$ & $1987-2001$ & -0.1 & & & & \\
\hline (Delay-adjusted) & $1975-1987$ & $1.0^{\mathrm{f}}$ & 1987-1995 & -0.5 & 1995-2001 & $0.9^{\mathrm{f}}$ & & \\
\hline Non-Hodgkin lymphoma & $1975-1991$ & $4.3^{\mathrm{f}}$ & $1991-2001$ & -0.1 & & & & \\
\hline (Delay-adjusted) & $1975-1991$ & $4.3^{\mathrm{f}}$ & $1991-2001$ & 0.1 & & & & \\
\hline Melanoma of the skin & $1975-1985$ & $5.6^{\mathrm{f}}$ & $1985-2001$ & $3.1^{\mathrm{f}}$ & & & & \\
\hline Oral cavity and pharynx & 1975-1993 & $-0.7^{\mathrm{f}}$ & 1993-2001 & $-2.8^{\mathrm{f}}$ & & & & \\
\hline Leukemia & $1975-1988$ & 0.2 & $1988-2001$ & $-0.7^{\mathrm{f}}$ & & & & \\
\hline (Delay-adjusted) & $1975-2001$ & 0.1 & & & & & & \\
\hline Kidney and renal pelvis & 1975-1987 & $2.3^{\mathrm{f}}$ & $1987-2001$ & $1.2^{\mathrm{f}}$ & & & & \\
\hline (Delay-adjusted) & $1975-2001$ & $1.8^{\mathrm{f}}$ & & & & & & \\
\hline Stomach & $1975-1989$ & $-1.2^{\mathrm{f}}$ & 1989-2001 & $-2.2^{\mathrm{f}}$ & & & & \\
\hline (Delay-adjusted) & 1975-1988 & $-1.1^{\mathrm{f}}$ & 1988-2001 & $-2.1^{\mathrm{f}}$ & & & & \\
\hline Pancreas & $1975-1978$ & -2.8 & 1978-2001 & $-0.5^{\mathrm{f}}$ & & & & \\
\hline (Delay-adjusted) & $1975-1993$ & $-0.8^{\mathrm{f}}$ & $1993-2001$ & 0.2 & & & & \\
\hline Liver and intrahepatic bile duct & $1975-1984$ & 1.7 & 1984-1999 & $4.5^{\mathrm{f}}$ & 1999-2001 & -4.9 & & \\
\hline (Delay-adjusted) & 1975-1984 & 1.7 & 1984-1999 & $4.6^{\mathrm{f}}$ & 1999-2001 & -2.7 & & \\
\hline Brain and other nervous system & 1975-1989 & $1.2^{\mathrm{f}}$ & 1989-2001 & -0.5 & & & & \\
\hline (Delay-adjusted) & 1975-1989 & $1.2^{\mathrm{f}}$ & $1989-2001$ & -0.3 & & & & \\
\hline Esophagus & $1975-2001$ & $0.8^{\mathrm{f}}$ & & & & & & \\
\hline (Delay-adjusted) & $1975-2001$ & $0.8^{\mathrm{f}}$ & & & & & & \\
\hline Larynx & $1975-1988$ & -0.2 & 1988-2001 & $-2.8^{\mathrm{f}}$ & & & & \\
\hline \multicolumn{9}{|l|}{ Top 15 for females } \\
\hline Breast & 1975-1980 & -0.4 & 1980-1987 & $3.7^{\mathrm{f}}$ & 1987-2001 & $0.4^{\mathrm{f}}$ & & \\
\hline (Delay-adjusted) & $1975-1980$ & -0.4 & $1980-1987$ & $3.7^{\mathrm{f}}$ & $1987-2001$ & $0.5^{\mathrm{f}}$ & & \\
\hline Lung and bronchus & $1975-1988$ & $4.6^{\mathrm{f}}$ & 1988-1998 & $1.3^{\mathrm{f}}$ & $1998-2001$ & $-2.3^{\mathrm{f}}$ & & \\
\hline (Delay-adjusted) & 1975-1982 & $5.5^{\mathrm{f}}$ & $1982-1990$ & $3.5^{\mathrm{f}}$ & 1990-1998 & $1.1^{\mathrm{f}}$ & 1998-2001 & -1.3 \\
\hline Colon and rectum & $1975-1985$ & $0.3^{\mathrm{f}}$ & $1985-1995$ & $-1.9^{\mathrm{f}}$ & 1995-1998 & 1.7 & 1998-2001 & $-2.8^{\mathrm{f}}$ \\
\hline (Delay-adjusted) & $1975-1985$ & $0.3^{f}$ & 1985-1995 & $-1.9^{\mathrm{f}}$ & 1995-1998 & 1.8 & 1998-2001 & $-2.3^{\mathrm{f}}$ \\
\hline Corpus and uterus, NOS & 1975-1979 & $-6.0^{\mathrm{f}}$ & 1979-1988 & $-1.7^{\mathrm{f}}$ & 1988-1998 & $0.6^{\mathrm{f}}$ & 1998-2001 & -1.5 \\
\hline Non-Hodgkin lymphoma & $1975-1988$ & $3.0^{\mathrm{f}}$ & 1988-1998 & $1.5^{\mathrm{f}}$ & $1998-2001$ & -1.6 & & \\
\hline (Delay-adjusted) & $1975-1990$ & $2.9^{\mathrm{f}}$ & 1990-2001 & $1.1^{\mathrm{f}}$ & & & & \\
\hline Ovary $^{\mathrm{e}}$ & $1975-1985$ & 0.2 & $1985-2001$ & $-0.8^{\mathrm{f}}$ & & & & \\
\hline (Delay-adjusted) $^{\mathrm{e}}$ & $1975-1985$ & 0.2 & $1985-2001$ & $-0.7^{\mathrm{f}}$ & & & & \\
\hline Melanoma of the skin & $1975-1981$ & $5.1^{\mathrm{f}}$ & $1981-2001$ & $2.2^{\mathrm{f}}$ & & & & \\
\hline Cervix uteri & $1975-1982$ & $-4.6^{\mathrm{f}}$ & $1982-1990$ & -0.2 & 1990-2001 & $-2.5^{\mathrm{f}}$ & & \\
\hline (Delay-adjusted) & $1975-1982$ & $-4.5^{\mathrm{f}}$ & $1982-1990$ & -0.2 & $1990-2001$ & $-2.4^{\mathrm{f}}$ & & \\
\hline Pancreas & $1975-1984$ & $1.4^{\mathrm{f}}$ & 1984-2001 & $-0.4^{\mathrm{f}}$ & & & & \\
\hline (Delay-adjusted) & $1975-1984$ & $1.3^{\mathrm{f}}$ & $1984-2001$ & $-0.3^{\mathrm{f}}$ & & & & \\
\hline & & & & & & & & tinued) \\
\hline
\end{tabular}


TABLE 1

(continued)

\begin{tabular}{|c|c|c|c|c|c|c|c|c|}
\hline & \multicolumn{8}{|c|}{ Joinpoint analyses (1975-2001) } \\
\hline & \multicolumn{2}{|c|}{ Trend 1} & \multicolumn{2}{|c|}{ Trend 2} & \multicolumn{2}{|c|}{ Trend 3} & \multicolumn{2}{|c|}{ Trend 4} \\
\hline & Years & $A P C^{d}$ & Years & $A P C^{d}$ & Years & $\mathrm{APC}^{\mathrm{d}}$ & Years & $A P C^{d}$ \\
\hline Leukemia & 1975-2001 & -0.1 & & & & & & \\
\hline (Delay-adjusted) & $1975-1999$ & $0.2^{\mathrm{f}}$ & 1999-2001 & 5.0 & & & & \\
\hline Thyroid & $1975-1981$ & -1.3 & $1981-1993$ & $2.1^{\mathrm{f}}$ & 1993-2001 & $4.5^{\mathrm{f}}$ & & \\
\hline Urinary bladder & $1975-2001$ & $0.2^{\mathrm{f}}$ & & & & & & \\
\hline (Delay-adjusted) & $1975-2001$ & $0.2^{\mathrm{f}}$ & & & & & & \\
\hline Kidney and renal pelvis & $1975-1992$ & $2.7^{\mathrm{f}}$ & 1992-2001 & $1.1^{\mathrm{f}}$ & & & & \\
\hline (Delay-adjusted) & $1975-2001$ & $2.3^{\mathrm{f}}$ & & & & & & \\
\hline Oral cavity and pharynx & $1975-1980$ & $2.6^{\mathrm{f}}$ & $1980-2001$ & $-1.0^{\mathrm{f}}$ & & & & \\
\hline Stomach & $1975-1996$ & $-1.9^{\mathrm{f}}$ & $1996-1999$ & 2.4 & 1999-2001 & -9.0 & & \\
\hline (Delay-adjusted) & $1975-1996$ & $-1.9^{\mathrm{f}}$ & $1996-1999$ & 2.5 & 1999-2001 & -8.3 & & \\
\hline
\end{tabular}

Source: Nine SEER areas (San Francisco-Oakland, Connecticut, Detroit-Metropolitan, Hawaii, Iowa, New Mexico, Seattle-Puget Sound, Utah, and Atlanta-Metropolitan).

SEER: Surveillance, Epidemiology, and End Results Program; APC: annual percent change; NOS: not otherwise specified.

a Joinpoint (JP) Regression Program, Version 2.7. Sept. 2003, National Cancer Institute.

${ }^{\mathrm{b}}$ The top 15 cancers were selected based on the sex-specific age-adjusted rate for 1992-2001 for all races combined.

${ }^{\mathrm{c}}$ Joinpoint analyses with up to three joinpoints are based on rates per 100,000 age-adjusted to the 2000 U.S. standard population.

${ }^{\mathrm{d}}$ Annual percent change based on rates that were age-adjusted to the 2000 U.S. standard population using joinpoint regression analysis.

${ }^{\mathrm{e}}$ All sites excludes myelodysplastic syndromes and borderline tumors; ovary excludes borderline tumors.

${ }^{\mathrm{f}}$ The annual percent change is statistically significantly different from zero (two-sided $P<0.05$ ).

panics/Latinos, for whom colorectal cancer ranked ahead of lung cancer (Table 3). The same three sites (with lung first and prostate second) were the leading causes of cancer death among men in each population, except in API men, in whom colon/rectum and liver cancers ranked second and third, respectively (Table 4). Among women, the three leading cancer sites in rank order for incidence in each racial and ethnic population were breast, colon/rectum, and lung, except in white women, in whom lung cancer ranked second (Table 3). The same three sites (with lung first and breast second) were the leading causes of cancer death among women in each population, except Hispanic/Latina women, whose death rates for breast cancer were higher than for cancer of the lung (Table 4).

Examination of short-term trends in incidence by race and ethnicity between 1992-2001 revealed that rates for lung and prostate cancer were decreasing among men in all populations, except API men (only lung decreasing); colorectal cancer rates were decreasing only for white and API men (Table 3). Among females, breast cancer incidence rates were increasing in white and API women and were decreasing among AI/AN women; lung cancer rates also were decreasing in AI/AN women and were stable for the other populations.

Incidence trends for other cancer sites also varied by race/ethnicity and sex (Table 3). Trends for many sites cannot be examined among AI/AN men and women because of small numbers; these sites are not noted in the results. Among men, declines in stomach cancer and nonepithelial skin cancers were observed in all populations; cancers of the oral cavity and larynx also were reported to decrease in all populations except among API men. Cervical cancer rates were reportedly decreasing for women of all racial and ethnic populations except AI/AN women (annual percent change $=-7.0 ; P>0.05)$. Thyroid cancer rates were increasing among white, black, and Hispanic/Latina women, and kidney cancer increased among women of all racial and ethnic populations except among AI/AN women.

Analyses of short-term mortality trends revealed that death rates decreased in men for cancers of the lung, prostate, and colon/rectum in each racial and ethnic population, except for the lung in AI/AN men and the colon/rectum in AI/AN and Hispanic/Latino men (Table 4). Among women, death rates decreased for colorectal cancer in white, black, and API women and for breast cancer in white, black and Hispanic/ Latina women, whereas rates increased for lung cancer in white women.

Mortality trends for cancer sites other than the top three sites also varied by race/ethnicity and sex (Table 4 ). Death rates for esophageal cancer increased among white men between 1992-2001 and decreased among black and Hispanic/Latino men. Stomach cancer death rates decreased in men and women of all racial and 
TABLE 2

U.S. Death Rate Trends with Joinpoint ${ }^{\mathrm{a}}$ Analyses for 1975 through 2001 for the Top 15 Cancers, ${ }^{\text {b }}$ All Races

\begin{tabular}{|c|c|c|c|c|c|c|c|c|}
\hline & \multicolumn{8}{|c|}{ Joinpoint analyses (1975-2001) ${ }^{c}$} \\
\hline & \multicolumn{2}{|c|}{ Trend 1} & \multicolumn{2}{|c|}{ Trend 2} & \multicolumn{2}{|c|}{ Trend 3} & \multicolumn{2}{|c|}{ Trend 4} \\
\hline & Years & $\mathrm{APC}^{\mathrm{d}}$ & Years & $\mathrm{APC}^{\mathrm{d}}$ & Years & $\mathrm{APC}^{\mathrm{d}}$ & Years & $\mathrm{APC}^{\mathrm{d}}$ \\
\hline \multicolumn{9}{|l|}{ All sites } \\
\hline Both sexes & 1975-1990 & $0.5^{\mathrm{e}}$ & 1990-1993 & -0.3 & 1993-2001 & $-1.1^{\mathrm{e}}$ & & \\
\hline Male & 1975-1979 & $1.0^{\mathrm{e}}$ & 1979-1990 & $0.3^{\mathrm{e}}$ & 1990-1993 & -0.4 & 1993-2001 & $-1.5^{\mathrm{e}}$ \\
\hline Female & 1975-1992 & $0.5^{\mathrm{e}}$ & 1992-2001 & $-0.8^{\mathrm{e}}$ & & & & \\
\hline \multicolumn{9}{|l|}{ Top 15 for males } \\
\hline Lung and bronchus & 1975-1982 & $1.8^{\mathrm{e}}$ & 1982-1991 & $0.4^{\mathrm{e}}$ & 1991-2001 & $-1.9^{\mathrm{e}}$ & & \\
\hline Prostate & 1975-1987 & $0.9^{\mathrm{e}}$ & 1987-1991 & $3.0^{\mathrm{e}}$ & 1991-1994 & -0.6 & 1994-2001 & $-4.1^{\mathrm{e}}$ \\
\hline Colon and rectum & 1975-1984 & -0.1 & 1984-1990 & $-1.4^{\mathrm{e}}$ & 1990-2001 & $-2.0^{\mathrm{e}}$ & & \\
\hline Pancreas & $1975-1986$ & $-0.8^{\mathrm{e}}$ & $1986-2001$ & $-0.3^{\mathrm{e}}$ & & & & \\
\hline Non-Hodgkin lymphoma & 1975-1996 & $2.5^{\mathrm{e}}$ & 1996-2001 & $-2.4^{\mathrm{e}}$ & & & & \\
\hline Leukemia & 1975-1995 & $-0.2^{\mathrm{e}}$ & 1995-2001 & $-0.8^{\mathrm{e}}$ & & & & \\
\hline Urinary bladder & $1975-1983$ & $-1.4^{\mathrm{e}}$ & $1983-1987$ & $-2.8^{\mathrm{e}}$ & 1987-1993 & 0.1 & 1993-2001 & $-0.7^{\mathrm{e}}$ \\
\hline Esophagus & 1975-1985 & $0.7^{\mathrm{e}}$ & 1985-1994 & $1.2^{\mathrm{e}}$ & 1994-2001 & $0.5^{\mathrm{e}}$ & & \\
\hline Stomach & 1975-1994 & $-2.1^{\mathrm{e}}$ & 1994-2001 & $-3.7^{\mathrm{e}}$ & & & & \\
\hline Liver and intrahepatic bile duct & $1975-1986$ & $1.6^{\mathrm{e}}$ & 1986-1995 & $4.0^{\mathrm{e}}$ & 1995-2001 & $1.4^{\mathrm{e}}$ & & \\
\hline Kidney and renal pelvis & $1975-1991$ & $1.1^{\mathrm{e}}$ & $1991-2001$ & -0.1 & & & & \\
\hline Brain and other nervous system & $1975-1977$ & 4.4 & 1977-1982 & -0.4 & $1982-1990$ & $1.5^{\mathrm{e}}$ & $1990-2001$ & $-0.7^{\mathrm{e}}$ \\
\hline Myeloma & 1975-1994 & $1.5^{\mathrm{e}}$ & $1994-2001$ & $-1.1^{\mathrm{e}}$ & & & & \\
\hline Oral cavity and pharynx & 1975-1993 & $-1.9^{\mathrm{e}}$ & 1993-2001 & $-3.0^{\mathrm{e}}$ & & & & \\
\hline Melanoma of the skin & 1975-1990 & $2.2^{\mathrm{e}}$ & 1990-2001 & 0.1 & & & & \\
\hline \multicolumn{9}{|l|}{ Top 15 for females } \\
\hline Lung and bronchus & 1975-1982 & $6.0^{\mathrm{e}}$ & 1982-1990 & $4.2^{\mathrm{e}}$ & 1990-1995 & $1.7^{\mathrm{e}}$ & $1995-2001$ & 0.2 \\
\hline Breast & $1975-1990$ & $0.4^{\mathrm{e}}$ & 1990-2001 & $-2.3^{\mathrm{e}}$ & & & & \\
\hline Colon and rectum & $1975-1984$ & $-1.0^{\mathrm{e}}$ & $1984-2001$ & $-1.8^{\mathrm{e}}$ & & & & \\
\hline Pancreas & $1975-1984$ & $0.8^{\mathrm{e}}$ & $1984-2001$ & 0.1 & & & & \\
\hline Ovary & $1975-1982$ & $-1.2^{\mathrm{e}}$ & $1982-1992$ & 0.3 & 1992-1998 & $-1.2^{\mathrm{e}}$ & 1998-2001 & 0.8 \\
\hline Non-Hodgkin lymphoma & 1975-1996 & $2.2^{\mathrm{e}}$ & 1996-2001 & $-2.5^{\mathrm{e}}$ & & & & \\
\hline Leukemia & 1975-1980 & 0.8 & 1980-2001 & $-0.4^{\mathrm{e}}$ & & & & \\
\hline Corpus and uterus, NOS & 1975-1991 & $-1.6^{\mathrm{e}}$ & 1991-2001 & -0.2 & & & & \\
\hline Brain and other nervous system & $1975-1992$ & $0.9^{\mathrm{e}}$ & $1992-2001$ & $-1.0^{\mathrm{e}}$ & & & & \\
\hline Stomach & 1975-1987 & $-2.8^{\mathrm{e}}$ & 1987-1990 & -0.5 & 1990-2001 & $-2.6^{\mathrm{e}}$ & & \\
\hline Myeloma & $1975-1993$ & $1.5^{\mathrm{e}}$ & $1993-2001$ & -0.4 & & & & \\
\hline Cervix uteri & $1975-1982$ & $-4.4^{\mathrm{e}}$ & $1982-1996$ & $-1.6^{\mathrm{e}}$ & $1996-2001$ & $-3.8^{\mathrm{e}}$ & & \\
\hline Liver and intrahepatic bile duct & 1975-1987 & $0.8^{\mathrm{e}}$ & 1987-1995 & $3.8^{\mathrm{e}}$ & $1995-2001$ & 0.3 & & \\
\hline Kidney and renal pelvis & 1975-1992 & $1.3^{\mathrm{e}}$ & 1992-2001 & -0.5 & & & & \\
\hline Urinary bladder & $1975-1977$ & 2.1 & 1977-1985 & $-2.2^{\mathrm{e}}$ & $1985-2001$ & $-0.4^{\mathrm{e}}$ & & \\
\hline \multicolumn{9}{|c|}{ Source: National Center for Health Statistics public-use data file for the total U.S. } \\
\hline \multicolumn{9}{|c|}{ APC: annual percent change; NOS: not otherwise specified. } \\
\hline \multicolumn{9}{|c|}{ a Joinpoint Regression Program, Version 2.7. Sept. 2003, National Cancer Institute. } \\
\hline \multicolumn{9}{|c|}{ ' ${ }^{\mathrm{b}}$ The top 15 cancers were selected based on the sex-specific age-adjusted rate for 1992-2001 for all races combined. } \\
\hline \multicolumn{9}{|c|}{ ' Joinpoint analyses with up to three joinpoints are based on rates per 100,000 age-adjusted to the 2000 U.S. standard population. } \\
\hline \multicolumn{9}{|c|}{ d Annual percent change based on rates that were age-adjusted to the 2000 U.S. standard population using joinpoint regression analysis. } \\
\hline${ }^{\mathrm{e}}$ The annual percent change is statistically & antly different $\mathrm{f}$ & (twh cid & & & & & & \\
\hline
\end{tabular}

ethnic populations except for Hispanic/Latina women and AI/AN men and women. Similarly, cancers of the oral cavity among men and women decreased in all populations, except for AI/AN men and women, API women, and Hispanic/Latina women. Finally, death rates for cancers of the gallbladder decreased among white, API, and Hispanic/
Latina women, and cervical cancer death rates decreased in all populations.

\section{Cancer in the white population}

Among women, white women had the highest cancer incidence rates for all cancer sites combined (Table 3). The incidence rates for cancers of the 
TABLE 3

SEER Incidence Rates and Trends for the Top 15 Cancers $^{\mathrm{a}}$ by Sex and Race/Ethnicity for 1992-2001

\begin{tabular}{|c|c|c|c|c|c|c|c|c|c|c|c|c|c|c|c|c|c|c|}
\hline \multirow[b]{2}{*}{ Sex/cancer site } & \multicolumn{3}{|c|}{ All races } & \multicolumn{3}{|c|}{ Whites } & \multicolumn{3}{|c|}{ Blacks } & \multicolumn{3}{|c|}{ API } & \multicolumn{3}{|c|}{ AI/AN } & \multicolumn{3}{|c|}{ Hispanics/Latinos ${ }^{\mathrm{b}}$} \\
\hline & Rank & Rate & APC & Rank & Rate & APC & Rank & Rate & APC & Rank & Rate & APC & Rank & Rate & APC & Rank & Rate & APC \\
\hline \multicolumn{19}{|l|}{ Male } \\
\hline All sites ${ }^{\mathrm{c}}$ & & 572.0 & $-1.6^{\mathrm{d}}$ & & 573.5 & $-1.5^{\mathrm{d}}$ & & 719.0 & $-1.9^{\mathrm{d}}$ & & 395.8 & $-1.3^{\mathrm{d}}$ & & 286.3 & $-3.9^{\mathrm{d}}$ & & 431.3 & $-1.2^{\mathrm{d}}$ \\
\hline Prostate & 1 & 180.3 & $-2.5^{\mathrm{d}}$ & 1 & 175.6 & $-2.6^{\mathrm{d}}$ & 1 & 284.6 & $-2.3^{\mathrm{d}}$ & 1 & 105.0 & -1.9 & 1 & 62.9 & $-7.9^{\mathrm{d}}$ & 1 & 143.0 & $-1.1^{\mathrm{d}}$ \\
\hline Lung and bronchus & 2 & 83.5 & $-2.3^{\mathrm{d}}$ & 2 & 82.2 & $-2.3^{\mathrm{d}}$ & 2 & 124.5 & $-2.6^{\mathrm{d}}$ & 2 & 61.6 & $-1.3^{\mathrm{d}}$ & 2 & 51.7 & $-4.3^{\mathrm{d}}$ & 3 & 47.9 & $-2.7^{\mathrm{d}}$ \\
\hline Colon and rectum & 3 & 64.5 & $-1.1^{\mathrm{d}}$ & 3 & 64.4 & $-1.2^{\mathrm{d}}$ & 3 & 72.9 & -0.6 & 3 & 56.9 & $-0.7^{\mathrm{d}}$ & 3 & 40.8 & -2.8 & 2 & 48.8 & 0.5 \\
\hline Urinary bladder & 4 & 36.1 & -0.1 & 4 & 39.7 & 0.0 & 5 & 20.2 & -0.3 & 7 & 16.3 & 0.1 & 8 & 8.9 & - & 5 & 18.9 & -0.6 \\
\hline Non-Hodgkin lymphoma & 5 & 23.7 & -0.5 & 5 & 24.8 & -0.5 & 7 & 18.8 & -1.5 & 6 & 16.7 & 0.3 & 7 & 9.4 & - & 4 & 19.4 & -1.4 \\
\hline Melanoma of the skin & 6 & 20.2 & $2.7^{\mathrm{d}}$ & 6 & 23.6 & $3.2^{\mathrm{d}}$ & 23 & 1.3 & - & 21 & 1.6 & 1.7 & 19 & 2.2 & - & 17 & 4.1 & $3.4^{\mathrm{d}}$ \\
\hline Oral cavity and pharynx & 7 & 16.7 & $-2.4^{\mathrm{d}}$ & 8 & 16.5 & $-2.2^{\mathrm{d}}$ & 4 & 21.4 & $-3.4^{\mathrm{d}}$ & 8 & 12.7 & -2.3 & 6 & 12.3 & $-7.8^{\mathrm{d}}$ & 11 & 10.6 & $-3.4^{\mathrm{d}}$ \\
\hline Leukemia & 8 & 16.3 & $-1.3^{\mathrm{d}}$ & 7 & 17.3 & $-1.3^{\mathrm{d}}$ & 11 & 13.0 & -1.2 & 10 & 9.8 & -0.1 & 12 & 5.3 & - & 9 & 11.8 & -0.9 \\
\hline Kidney and renal pelvis & 9 & 15.3 & $1.3^{\mathrm{d}}$ & 9 & 15.7 & $1.4^{\mathrm{d}}$ & 8 & 18.1 & 1.9 & 11 & 8.8 & 0.4 & 4 & 15.0 & $-5.9^{\mathrm{d}}$ & 7 & 14.4 & 1.4 \\
\hline Stomach & 10 & 13.3 & $-2.3^{\mathrm{d}}$ & 11 & 11.5 & $-2.3^{\mathrm{d}}$ & 6 & 20.0 & $-2.6^{\mathrm{d}}$ & 4 & 23.5 & $-3.5^{\mathrm{d}}$ & 5 & 14.3 & - & 6 & 18.8 & $-2.8^{\mathrm{d}}$ \\
\hline Pancreas & 11 & 12.7 & -0.4 & 10 & 12.4 & 0.0 & 9 & 17.9 & $-2.0^{\mathrm{d}}$ & 9 & 10.8 & $-2.9^{\mathrm{d}}$ & 10 & 7.6 & - & 10 & 10.7 & -1.3 \\
\hline Liver and intrahepatic bile duct & 12 & 8.5 & $3.2^{\mathrm{d}}$ & 15 & 6.7 & $3.2^{\mathrm{d}}$ & 14 & 10.7 & $4.6^{\mathrm{d}}$ & 5 & 20.7 & 0.8 & 9 & 8.7 & - & 8 & 13.1 & 1.9 \\
\hline Brain and other nervous system & 13 & 7.7 & -0.7 & 12 & 8.5 & -0.5 & 16 & 4.7 & 0.1 & 14 & 4.2 & -0.9 & 15 & 3.0 & - & 14 & 5.7 & 0.4 \\
\hline Esophagus & 14 & 7.6 & 0.5 & 13 & 7.3 & $1.9^{\mathrm{d}}$ & 13 & 12.6 & $-5.8^{\mathrm{d}}$ & 12 & 5.2 & -1.3 & 11 & 5.7 & - & 13 & 6.0 & -0.4 \\
\hline Larynx & 15 & 7.3 & $-3.4^{\mathrm{d}}$ & 14 & 7.2 & $-3.3^{\mathrm{d}}$ & 12 & 12.9 & $-2.9^{\mathrm{d}}$ & 16 & 3.5 & -2.8 & 20 & 2.0 & - & 15 & 5.7 & $-1.5^{\mathrm{d}}$ \\
\hline Myeloma & 16 & 7.0 & -1.0 & 16 & 6.7 & -0.7 & 10 & 13.3 & -1.7 & 13 & 4.2 & -2.1 & 13 & 4.1 & - & 12 & 6.4 & -1.8 \\
\hline Other nonepithelial skin ${ }^{\mathrm{e}}$ & 17 & 6.3 & $-13.3^{\mathrm{d}}$ & 17 & 6.5 & $-14.2^{\mathrm{d}}$ & 15 & 6.9 & $-10.4^{\mathrm{d}}$ & 20 & 2.0 & $-9.9^{\mathrm{d}}$ & 18 & 2.4 & - & 16 & 5.4 & $-19.1^{\mathrm{d}}$ \\
\hline Thyroid & 19 & 3.6 & $2.4^{\mathrm{d}}$ & 19 & 3.8 & $2.8^{\mathrm{d}}$ & 20 & 2.1 & 0.8 & 15 & 3.8 & 0.7 & 21 & 1.9 & - & 21 & 2.8 & 1.8 \\
\hline Gallbladder & 29 & 0.9 & -2.2 & 31 & 0.8 & -1.7 & 30 & 0.8 & - & 22 & 1.4 & -5.7 & 14 & 3.0 & - & 25 & 1.3 & -0.2 \\
\hline \multicolumn{19}{|l|}{ Female } \\
\hline All sites ${ }^{\mathrm{c}}$ & & 411.8 & 0.1 & & 425.1 & 0.3 & & 401.9 & -0.4 & & 300.0 & 0.2 & & 228.7 & $-1.7^{\mathrm{d}}$ & & 309.4 & -0.1 \\
\hline Breast & 1 & 132.5 & $0.6^{\mathrm{d}}$ & 1 & 138.3 & $0.8^{\mathrm{d}}$ & 1 & 120.3 & -0.3 & 1 & 92.2 & $1.7^{\mathrm{d}}$ & 1 & 60.4 & $-3.7^{\mathrm{d}}$ & 1 & 88.3 & 0.7 \\
\hline Lung and bronchus & 2 & 49.2 & -0.2 & 2 & 51.3 & -0.2 & 3 & 53.7 & 0.5 & 3 & 28.3 & -0.1 & 3 & 25.9 & $-3.1^{\mathrm{d}}$ & 3 & 24.4 & -1.5 \\
\hline Colon and rectum & 3 & 46.6 & -0.5 & 3 & 46.1 & -0.6 & 2 & 56.1 & 0.0 & 2 & 39.0 & -0.4 & 2 & 32.1 & -0.9 & 2 & 32.5 & -0.1 \\
\hline Corpus and uterus, NOS & 4 & 24.5 & -0.1 & 4 & 26.1 & -0.1 & 4 & 18.0 & 0.9 & 4 & 16.8 & $1.4^{\mathrm{d}}$ & 4 & 9.9 & - & 5 & 16.5 & 0.5 \\
\hline Non-Hodgkin lymphoma & 5 & 15.4 & $0.8^{\mathrm{d}}$ & 5 & 16.2 & $0.8^{\mathrm{d}}$ & 7 & 10.8 & $2.9^{\mathrm{d}}$ & 7 & 11.1 & 1.3 & 10 & 6.9 & - & 6 & 13.3 & 0.6 \\
\hline Ovary $^{\mathrm{c}}$ & 6 & 14.2 & $-1.0^{\mathrm{d}}$ & 7 & 15.1 & $-0.8^{\mathrm{d}}$ & 8 & 10.3 & -1.6 & 9 & 10.2 & -0.6 & 5 & 9.2 & - & 7 & 11.7 & -1.0 \\
\hline Melanoma of the skin & 7 & 13.0 & $2.5^{\mathrm{d}}$ & 6 & 15.6 & $3.2^{\mathrm{d}}$ & 29 & 0.8 & - & 21 & $\begin{array}{r}1.3 \\
\end{array}$ & 4.5 & 19 & 1.9 & - & 17 & 4.1 & $3.3^{\mathrm{d}}$ \\
\hline Cervix uteri & 8 & 9.9 & $-2.6^{\mathrm{d}}$ & 12 & 9.4 & $-2.2^{\mathrm{d}}$ & 6 & 12.8 & $-3.1^{\mathrm{d}}$ & 8 & 10.9 & $-4.7^{\mathrm{d}}$ & 9 & 6.9 & -7.0 & 4 & 17.7 & $-3.3^{\mathrm{d}}$ \\
\hline Pancreas & 9 & 9.9 & -0.6 & 11 & 9.6 & -0.6 & 5 & 14.7 & $-2.5^{\mathrm{d}}$ & 10 & 8.2 & $2.3^{\mathrm{d}}$ & 8 & 7.1 & - & 10 & 9.3 & -0.4 \\
\hline Leukemia & 10 & 9.6 & $-0.8^{\mathrm{d}}$ & 8 & 10.1 & -0.5 & 12 & 8.1 & -0.5 & 12 & 6.3 & $-2.9^{\mathrm{d}}$ & 13 & 4.4 & - & 12 & 7.6 & -1.3 \\
\hline Thyroid & 11 & $\begin{array}{l}9.0 \\
9.5\end{array}$ & $4.3^{\mathrm{d}}$ & 10 & $\begin{array}{r}0.1 \\
9.8\end{array}$ & $4.8^{\mathrm{d}}$ & 15 & 5.2 & $3.8^{\mathrm{d}}$ & 6 & 11.4 & 1.3 & 12 & 5.8 & 2.6 & 9 & 9.5 & $2.5^{\mathrm{d}}$ \\
\hline Urinary bladder & 12 & 9.2 & $-0.5^{\mathrm{d}}$ & 9 & 9.9 & $-0.4^{\mathrm{d}}$ & 13 & 7.5 & 0.3 & 14 & 4.4 & 0.2 & 18 & 2.1 & - & 14 & 5.1 & -1.1 \\
\hline Kidney and renal pelvis & 13 & 7.6 & $1.5^{\mathrm{d}}$ & 13 & 7.8 & $1.4^{\mathrm{d}}$ & 11 & 9.0 & $2.8^{\mathrm{d}}$ & 15 & 4.1 & $3.1^{\mathrm{d}}$ & 6 & 8.1 & - & 11 & 7.7 & $3.0^{\mathrm{d}}$ \\
\hline Oral cavity and pharynx & 14 & 6.7 & $-1.4^{\mathrm{d}}$ & 14 & 6.7 & $-1.5^{\mathrm{d}}$ & 14 & 6.5 & -2.1 & 13 & 5.8 & -0.2 & 14 & 4.0 & - & 18 & 4.0 & -1.5 \\
\hline Stomach & 15 & 6.2 & -0.9 & 16 & 5.1 & -1.3 & 10 & 9.7 & 0.0 & 5 & 13.2 & $-2.7^{\mathrm{d}}$ & 7 & 7.6 & - & 8 & 10.2 & -1.2 \\
\hline Brain and other nervous system & 16 & 5.4 & -0.6 & 15 & 5.9 & -0.3 & 18 & 3.5 & -0.9 & 16 & 2.9 & $-3.6^{\mathrm{d}}$ & 20 & 1.7 & - & 15 & 4.5 & -0.1 \\
\hline Myeloma & 17 & 4.7 & -0.6 & 17 & 4.3 & -0.7 & 9 & 10.3 & -1.6 & 17 & 2.8 & 2.7 & 16 & 3.1 & - & 16 & 4.4 & -0.7 \\
\hline Liver and intrahepatic bile duct & 18 & 3.2 & $3.3^{\mathrm{d}}$ & 19 & 2.6 & $3.9^{\mathrm{d}}$ & 17 & 3.6 & $\begin{array}{l}1.0 \\
1.9\end{array}$ & 11 & $\begin{array}{l}.0 \\
7.8\end{array}$ & $\begin{array}{r}2.1 \\
-0.9\end{array}$ & 11 & $\begin{array}{l}.1 \\
5.8\end{array}$ & - & 13 & $\begin{array}{l}4.4 \\
5.3\end{array}$ & $4.3^{\mathrm{d}}$ \\
\hline Gallbladder & 23 & 1.6 & $-1.8^{\mathrm{d}}$ & 23 & 1.6 & -1.6 & 24 & 1.6 & -1.5 & 20 & 1.7 & -3.8 & 15 & 3.8 & - & 19 & 4.0 & $-4.2^{\mathrm{d}}$ \\
\hline
\end{tabular}

Source: 12 SEER areas (San Francisco-Oakland SMSA, Connecticut, Detroit-Metropolitan, Hawaii, Iowa, New Mexico, Seattle-Puget Sound, Utah, Atlanta-Metropolitan, San Jose-Monterey, Los Angeles, and Alaska). SEER: Surveillance, Epidemiology, and End Results Program; APC: annual percent change, API: Asian/Pacific Islander; AI/AN: American Indian/Alaska Native; NOS: not otherwise specified.

${ }^{a}$ Cancers are sorted in descending order according to sex-specific rates for all races. More than 15 cancers may appear under male and female to include the top 15 cancers in every racial and ethnic group.

${ }^{\mathrm{b}}$ Data for Hispanics/Latinos excludes cases diagnosed in Detroit and Hawaii.

${ }^{\mathrm{c}}$ All sites excludes myelodysplastic syndromes and borderline tumors; ovary excludes borderline tumors.

${ }^{\mathrm{d}}$ The annual percent change is statistically significantly different from zero (two-sided $P<0.05$ ).

${ }^{\text {e }}$ For other nonepithelial skin for males, $70 \%$ is Kaposi sarcoma, $68 \%$ for white males and $86 \%$ for black males.

-Statistic could not be calculated. The annual percent change is based on fewer than 10 cases for at least 1 year within the time interval. 
TABLE 4

U.S. Death Rates and Trends for the Top 15 Cancers $^{\mathrm{a}}$ by Sex and Race/Ethnicity for 1992-2001

\begin{tabular}{|c|c|c|c|c|c|c|c|c|c|c|c|c|c|c|c|c|c|c|}
\hline \multirow[b]{2}{*}{ Sex/cancer site } & \multicolumn{3}{|c|}{ All races } & \multicolumn{3}{|c|}{ Whites } & \multicolumn{3}{|c|}{ Blacks } & \multicolumn{3}{|c|}{ API } & \multicolumn{3}{|c|}{ AI/AN } & \multicolumn{3}{|c|}{ Hispanics/Latinos $^{\mathrm{b}}$} \\
\hline & Rank & Rate & APC & Rank & Rate & APC & Rank & Rate & APC & Rank & Rate & APC & Rank & Rate & APC & Rank & Rate & $\mathrm{APC}$ \\
\hline \multicolumn{19}{|l|}{ Male } \\
\hline All sites & & 260.6 & $-1.5^{\mathrm{c}}$ & & 254.3 & $-1.4^{\mathrm{c}}$ & & 364.7 & $-1.9^{\mathrm{c}}$ & & 157.9 & $-1.9^{c}$ & & 168.1 & -0.7 & & 177.1 & $-0.8^{\mathrm{c}}$ \\
\hline Lung and bronchus & 1 & 81.6 & $-1.9^{\mathrm{c}}$ & 1 & 80.0 & $-1.7^{\mathrm{c}}$ & 1 & 110.7 & $-2.4^{\mathrm{c}}$ & 1 & 41.6 & $-1.7^{\mathrm{c}}$ & 1 & 51.0 & -1.8 & 1 & 40.9 & $-1.4^{c}$ \\
\hline Prostate & 2 & 34.6 & $-3.6^{\mathrm{c}}$ & 2 & 31.8 & $-3.7^{\mathrm{c}}$ & 2 & 74.9 & $-2.3^{\mathrm{c}}$ & 4 & 14.7 & $-4.8^{\mathrm{c}}$ & 2 & 22.3 & $-3.9^{\mathrm{c}}$ & 2 & 25.0 & $-2.3^{\mathrm{c}}$ \\
\hline Colon and rectum & 3 & 26.6 & $-2.1^{\mathrm{c}}$ & 3 & 26.1 & $-2.2^{\mathrm{c}}$ & 3 & 35.0 & $-0.8^{\mathrm{c}}$ & 2 & 16.4 & $-1.8^{\mathrm{c}}$ & 3 & 16.1 & 2.5 & 3 & 18.1 & -0.1 \\
\hline Pancreas & 4 & 12.3 & $-0.3^{\mathrm{c}}$ & 4 & 12.0 & -0.1 & 4 & 16.6 & $-1.6^{c}$ & 6 & 8.5 & $-2.2^{\mathrm{c}}$ & 7 & 6.2 & 1.8 & 6 & 9.5 & -0.5 \\
\hline dgkin lymphoma & 5 & 10.5 & -0.3 & 5 & 10.9 & -0.3 & 11 & 7.5 & -0.3 & 7 & 6.8 & -1.4 & 8 & 4 & $5.0^{\mathrm{c}}$ & 7 & 8.1 & -0.4 \\
\hline Leukemia & 6 & 10.4 & $-0.7^{\mathrm{c}}$ & 6 & 10.7 & $-0.6^{\mathrm{c}}$ & 7 & 9.4 & $-1.1^{\mathrm{c}}$ & 8 & 5.5 & -1.2 & 9 & 5.2 & -3.9 & 8 & 6.7 & 0.7 \\
\hline Urinary bladder & 7 & 7.7 & $-0.6^{\mathrm{c}}$ & 7 & 8.0 & $-0.5^{\mathrm{c}}$ & 13 & 5.9 & $-2.2^{\mathrm{c}}$ & 11 & 2.9 & 0.3 & 13 & 2.5 & 1.1 & 11 & 4.2 & -0.8 \\
\hline Esophagus & 8 & 7.6 & $0.7^{\mathrm{c}}$ & 8 & 7.1 & $1.7^{\mathrm{c}}$ & 6 & 13.2 & $-4.3^{\mathrm{c}}$ & 10 & 3.7 & -3.1 & 10 & 4.7 & 0.9 & 10 & 4.5 & $-1.2^{\mathrm{c}}$ \\
\hline Stomach & 9 & 7.2 & $-3.4^{\mathrm{c}}$ & 9 & 6.4 & $-3.6^{\mathrm{c}}$ & 5 & 14.3 & $-2.9^{\mathrm{c}}$ & 5 & 13.0 & $-3.5^{\mathrm{c}}$ & 5 & 7.6 & -1.2 & 5 & 10.1 & $-2.0^{\mathrm{c}}$ \\
\hline Liver and intrahepatic bile duct & 10 & 6.4 & $2.0^{\mathrm{c}}$ & 12 & 5.8 & $2.1^{\mathrm{c}}$ & 9 & 9.1 & 1.1 & 3 & 15.9 & -0.8 & 4 & 7.7 & 2.5 & 4 & 10.1 & $1.7^{\mathrm{c}}$ \\
\hline Kidney and renal pelvis & 11 & 6.2 & -0.1 & 10 & 6.2 & -0.1 & 12 & 6.2 & 0.5 & 12 & 2.7 & 0.8 & 6 & 6.7 & -0.4 & 9 & 5.5 & 0.8 \\
\hline Brain and other nervous system & 12 & 5.7 & $-0.7^{\mathrm{c}}$ & 11 & 6.1 & $-0.6^{\mathrm{c}}$ & 15 & 3.3 & $-0.8^{\mathrm{c}}$ & 13 & 2.3 & 1.8 & 14 & 2.5 & 3.0 & 13 & 3.5 & 0.6 \\
\hline Myeloma & 13 & 4.8 & -0.6 & 13 & 4.5 & -0.4 & 8 & 9.2 & $-1.0^{\mathrm{c}}$ & 14 & 2.2 & -1.8 & 12 & 3.5 & -1.8 & 12 & 3.8 & 0.5 \\
\hline Oral cav & 14 & 4.6 & $-2.8^{\mathrm{c}}$ & 15 & 4.2 & $-2.5^{\mathrm{c}}$ & 10 & 8.4 & $-4.4^{\mathrm{c}}$ & 9 & 3.9 & $-2.5^{\mathrm{c}}$ & 11 & .7 & -1.0 & 14 & 3.3 & $-4.2^{\mathrm{c}}$ \\
\hline Melanon & 15 & 3.9 & 0.0 & 14 & 4.4 & 0.2 & 23 & 0.5 & -0.7 & 20 & 0.5 & -3.8 & 19 & 8 & 0.3 & 17 & 1.1 & 0.5 \\
\hline Larynx & 16 & 2.7 & $-2.3^{\mathrm{c}}$ & 16 & 2.5 & $-2.2^{\mathrm{c}}$ & 14 & 5.8 & $-2.8^{c}$ & 16 & 1.0 & 0 & 15 & 2.0 & 5.7 & 15 & 2.3 & -2.0 \\
\hline Soft tissue including heart & 17 & 1.6 & -0.9 & 17 & 1.6 & -0.9 & 16 & 1.6 & 0.3 & 15 & 1.1 & -3.4 & 18 & 0.9 & -7.0 & 16 & 1.2 & -1.7 \\
\hline \multicolumn{19}{|l|}{ Female } \\
\hline All sites & & 169.9 & $-0.7^{\mathrm{c}}$ & & 168.6 & $-0.7^{\mathrm{c}}$ & & 200.1 & $-0.8^{\mathrm{c}}$ & & 103.0 & $-1.1^{\mathrm{c}}$ & & 115.1 & -0.4 & & 112.4 & $-0.3^{\mathrm{c}}$ \\
\hline Lung and & 1 & 40.2 & $0.6^{c}$ & 1 & 41.0 & $0.7^{\mathrm{c}}$ & 1 & 39.2 & 0.4 & 1 & 19.2 & 0.1 & 1 & 26.2 & 1.0 & 2 & 14.8 & 0.1 \\
\hline Breast & 2 & 28.8 & $-2.4^{\mathrm{c}}$ & 2 & 28.3 & $-2.6^{\mathrm{c}}$ & 2 & 36.4 & $-1.2^{\mathrm{c}}$ & 2 & 12.9 & -0.9 & 2 & 14.5 & -1.8 & 1 & 17.9 & $-1.8^{\mathrm{c}}$ \\
\hline Colon and & 3 & 18.5 & $-1.7^{\mathrm{c}}$ & 3 & 18.0 & $-1.8^{\mathrm{c}}$ & 3 & 24.9 & $-0.7^{\mathrm{c}}$ & 3 & 11.4 & $-2.1^{\mathrm{c}}$ & 3 & 12.0 & -0.8 & 3 & 11.5 & 0.2 \\
\hline Pancreas & 4 & 9.2 & -0.1 & 5 & 8.9 & 0.0 & 4 & 12.9 & $-0.7^{\mathrm{c}}$ & 5 & 6.7 & 0.6 & 4 & 6.0 & -0.9 & 4 & 7.5 & 0.1 \\
\hline Ovary & 5 & 9. & $-0.6^{c}$ & 4 & 9.3 & $-0.5^{\mathrm{c}}$ & 5 & 7.6 & $-0.9^{c}$ & 7 & 4.7 & -0.5 & 5 & 5.1 & -0.1 & 5 & 6.3 & -0.6 \\
\hline Non-Hc & 6 & 6. & -0.5 & 6 & 7.2 & -0.5 & 11 & 4.5 & -0.2 & 8 & 4.2 & -0.6 & 8 & 4.0 & $5.1^{\mathrm{c}}$ & 7 & 5.3 & 0.1 \\
\hline Leukemia & 7 & 6. & $-0.5^{\mathrm{c}}$ & 7 & 6.1 & $-0.4^{\mathrm{c}}$ & 10 & 5.5 & $-0.8^{\mathrm{c}}$ & 9 & 3.4 & -1.4 & 10 & 3.4 & -1.2 & 9 & 4.4 & -0.4 \\
\hline Corpus and & 8 & 4.1 & -0.2 & 9 & 3.9 & -0.2 & 6 & 7.0 & -0.1 & 11 & 2.2 & 0.4 & 13 & 2.5 & -1.8 & 11 & 3.2 & 0.4 \\
\hline Brain and other nervous system & 9 & 3.8 & $-1.1^{\mathrm{c}}$ & 8 & 4.1 & $-1.0^{\mathrm{c}}$ & 16 & 2.3 & -0.5 & 12 & 1.6 & -3.1 & 15 & 1.6 & 2.1 & 13 & 2.5 & 0.8 \\
\hline Stomach & 10 & 3.5 & $-2.6^{\mathrm{c}}$ & 10 & 3.0 & $-2.8^{\mathrm{c}}$ & 7 & 6.7 & $-2.3^{\mathrm{c}}$ & 4 & 7.7 & $-3.6^{\mathrm{c}}$ & 6 & 4.1 & -1.4 & 6 & 5.5 & -1.6 \\
\hline Myeloma & 11 & 3.2 & -0.3 & 11 & 2.9 & -0.3 & 8 & 6.6 & -0.1 & 13 & 1.5 & 0.9 & 12 & 2.7 & 3.1 & 12 & 2.7 & 1.6 \\
\hline Cervix uter & 12 & 3.1 & $-3.0^{\mathrm{c}}$ & 13 & 2.7 & $-2.5^{\mathrm{c}}$ & 9 & 6.3 & $-5.1^{\mathrm{c}}$ & 10 & 3.0 & $-2.7^{\mathrm{c}}$ & 11 & 3.2 & $-5.9^{\mathrm{c}}$ & 10 & 3.9 & $-2.9^{c}$ \\
\hline Liver and intra & 13 & 2.9 & $1.2^{\mathrm{c}}$ & 14 & 2.7 & $1.1^{\mathrm{c}}$ & 12 & 3.7 & 1.1 & 6 & 6.6 & -0.4 & 7 & 4.1 & 2.5 & 8 & 4.8 & $2.4^{\mathrm{c}}$ \\
\hline d renal pelvis & 14 & 2.8 & -0.4 & 12 & 2.9 & -0.4 & 15 & 2.8 & -0.1 & 15 & 1.2 & 1.6 & 9 & 3.4 & -1.7 & 14 & 2.5 & -0.6 \\
\hline Urinary bladder & 15 & 2.3 & $-0.6^{\mathrm{c}}$ & 15 & 2.3 & -0.5 & 14 & 3.0 & -1.0 & 16 & 1.1 & -3.0 & 18 & 1.0 & -3.0 & 16 & 1.3 & 0.4 \\
\hline Esophagus & 17 & 1.8 & 0.0 & 18 & 1.6 & $0.8^{\mathrm{c}}$ & 13 & 3.5 & $-3.3^{\mathrm{c}}$ & 18 & 0.9 & -1.9 & 17 & 1.1 & 7.8 & 18 & 0.9 & 2.2 \\
\hline Oral cavity a & 18 & 1.7 & $-2.5^{\mathrm{c}}$ & 17 & 1.7 & $-2.3^{\mathrm{c}}$ & 17 & 2.2 & $-3.3^{c}$ & 14 & 1.4 & -2.7 & 16 & 1.2 & -2.6 & 19 & 0.9 & 0.0 \\
\hline Gallbladder & 20 & 1.0 & $-2.4^{\mathrm{c}}$ & 20 & 0.9 & $-2.5^{\mathrm{c}}$ & 19 & 1.0 & -1.0 & 17 & 1.1 & $-5.8^{\mathrm{c}}$ & 14 & 1.8 & -2.3 & 15 & 1.9 & $-2.7^{c}$ \\
\hline
\end{tabular}

Source: National Center for Health Statistics public-use data file for the total U.S.

APC: annual percent change; API: Asian/Pacific Islander; AI/AN: American Indian/Alaska Native; NOS: not otherwise specified.

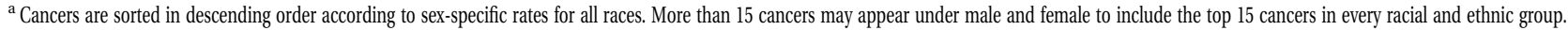

${ }^{\mathrm{b}}$ Data for Hispanics/Latinos excludes Connecticut, Maine, Maryland, New Hampshire, New York, North Dakota, Oklahoma, and Vermont.

${ }^{\mathrm{c}}$ The annual percent change is statistically significantly different from zero (two-sided $P<0.05$ ).

-Statistic could not be calculated. The annual percent change is based on fewer than 10 cases for at least 1 year within the time interval.

female breast and bladder, NHL, melanoma, leukemia, brain cancer, cancer of the corpus uterus (corpus), cancer of the ovary, and cancer of the oral cavity (females only) were higher in white men and women than in other racial and ethnic populations, with melanoma rates being substantially higher. The rate of bladder cancer in white men (39.7 cases per
100,000 men) was nearly twice that of black men (20.2 cases). Of the top 15 causes of cancer deaths in white men and women, rates of lung cancer (women only), cancer of the ovary, bladder cancer (men only), NHL, leukemia, brain cancer, and melanoma were higher than in all other racial and ethnic populations (Table 4). 


\section{Cancer in the black population}

Among men, black men had the highest cancer incidence and death rates for all cancer sites combined and for cancers of the prostate, lung, colon/rectum, oral cavity, stomach (mortality only), kidney (incidence only), pancreas, esophagus, larynx, and myeloma (Tables 3 and 4). The age-adjusted rate of prostate cancer incidence in black men was $62 \%$ higher, and the death rate was more than twice that of white men, who have the second highest incidence and death rates of all racial and ethnic populations studied. Black women had the highest death rates for all sites combined and the highest incidence and death rates for the following sites: breast (mortality only), colon/rectum, lung (incidence only), kidney (incidence only), oral cavity (mortality only), pancreas, esophagus, myeloma, corpus (mortality only), cervix uteri (mortality only), and bladder (mortality only). The incidence and death rates for myeloma in black men and women were approximately double the rates for white men and women. Female breast cancer death rates were nearly $30 \%$ higher in the black population than in the white population, despite lower incidence rates.

\section{Cancer in the API population}

The incidence rates for cancers of the stomach, liver, and thyroid (women only) were higher in API populations than in other racial and ethnic populations. Cancer death rates were higher for cancers of the liver and stomach (women only). The liver cancer incidence rate for males was 20.7 compared with 13.1 for Hispanics/ Latinos, the population with the next highest rate.

\section{Cancer in the Al/AN population}

The death rate for kidney cancer in AI/AN men and women was higher than in other racial and ethnic populations. Although death rates from cancers of the liver and stomach among AI/AN men were lower than for other populations (with the exception of white men), these sites had ranks of 4 and 5 , respectively, for cancer mortality among AI/AN men. Similarly, although the rates of ovarian cancer incidence in AI/AN women were lower than in other populations, ovarian cancer was the fifth most common cancer type in $\mathrm{AI} / \mathrm{AN}$ women, the highest ranking for ovarian cancer in any racial or ethnic population.

\section{Cancer in the Hispanic/Latino population}

The incidence rates for cancers of the cervix uteri and gallbladder and the death rate for cancer of the gallbladder were higher in Hispanic/Latina women than among other racial and ethnic populations. The incidence rate for cancer of cervix uteri in this population
(17.7) ranked 4th and was $38 \%$ higher than the rate for black women (12.8), the population with the next highest rate.

\section{Changes in Survival Rates over Time}

The absolute and proportional changes in survival for cancer of all sites combined, all ages, was substantially greater for men than for women, although the current survival rates generally were similar (Table 5). In men, cancers that demonstrated a large absolute gain $(\geq 10 \%)$ in survival were all sites combined, cancer of the prostate, colon/rectum cancer, NHL, melanoma, leukemia, and kidney cancer; survival for cancers of the bladder, oral cavity, stomach, liver, brain, and esophagus showed gains of 5-9.9\%. In women, cancers that demonstrated a large absolute gain $(\geq 10 \%)$ in survival were cancer of the colon/rectum, NHL, kidney cancer, and breast cancer; survival for cancer of all cancers combined, bladder cancer, melanoma, oral cavity cancer, leukemia, stomach cancer, brain cancer, cancer of the esophagus, and cancer of the ovary demonstrated gains of 5-9.9\%. Improvement in survival was limited for the most fatal cancers in adults (a 5-year survival rate of $<20 \%$ ), which included cancers of the lung, pancreas, liver, and esophagus. These cancers are characterized by a late stage at the time of diagnosis and/or relatively poor survival even when diagnosed at localized stage (Table 6). Several sites with high survival rates (larynx and thyroid) and cancers of the corpus and cervix uteri showed little or no gain. Between 1975-1979 and 1995-2000, survival also improved for several less common cancers not listed in Table 5, including testicular cancer (85-96\%) and Hodgkin lymphoma (74-85\%).

Survival rates for childhood cancers demonstrated some of the largest improvements, with an absolute survival increase of $20 \%$ in boys and $13 \%$ in girls between 1975-1979 and 1995-2000 (Table 5). The 5-year relative survival rates in 1995-2000 varied according to type of cancer, from $<50 \%$ for acute myeloid leukemia in boys to $>95 \%$ for Hodgkin lymphoma in both boys and girls.

\section{Stage-Specific Survival and Stage Distributions and Geographic Variations}

For most specific cancers, patients diagnosed at an earlier stage of disease had higher 5-year survival rates than those diagnosed at more advanced stages in the 9 SEER areas (Table 6). There were 9 cancer sites in which $\geq 50 \%$ of cases were diagnosed at a localized stage, including the prostate, urinary bladder, melanoma, kidney, larynx, thyroid, breast, corpus, and cervix uteri. In contrast, the majority of lung (53\%) and ovarian (68\%) cancers were diagnosed at a distant stage. 
TABLE 5

Trends in SEER 5-Year Relative Survival Rates ${ }^{a}$ for the Top 15 Cancers $^{\mathrm{b}}$ for All Ages and Childhood Cancers, by Sex, 1975-1979 to $1995-2000$

\begin{tabular}{|c|c|c|c|c|c|c|c|c|}
\hline \multirow[b]{3}{*}{ Cancer site } & \multicolumn{4}{|c|}{ Male } & \multicolumn{4}{|c|}{ Female } \\
\hline & \multicolumn{2}{|c|}{ Survival rate (\%) } & \multicolumn{2}{|c|}{ Change (\%) } & \multicolumn{2}{|c|}{ Survival rate (\%) } & \multicolumn{2}{|c|}{ Change (\%) } \\
\hline & $1975-1979$ & $1995-2000$ & Absolute $^{c}$ & Proportional $^{\mathbf{c}}$ & $1975-1979$ & $1995-2000$ & Absolute $^{c}$ & Proportional $^{\mathrm{c}}$ \\
\hline \multicolumn{9}{|l|}{ All ages } \\
\hline All sites ${ }^{\mathrm{d}}$ & 42.7 & $64.0^{\mathrm{e}}$ & 21.3 & 49.9 & 56.6 & $64.3^{\mathrm{e}}$ & 7.7 & 13.6 \\
\hline Prostate & 70.0 & $99.3^{\mathrm{e}}$ & 29.3 & 41.9 & - & - & - & - \\
\hline Lung and bronchus & 11.6 & $13.6^{\mathrm{e}}$ & 2.0 & 17.2 & 16.6 & $17.2^{\mathrm{e}}$ & 0.6 & 3.6 \\
\hline Colon and rectum & 50.3 & $63.7^{\mathrm{e}}$ & 13.4 & 26.6 & 52.3 & $63.1^{\mathrm{e}}$ & 10.8 & 20.7 \\
\hline Urinary bladder & 75.7 & $83.7^{\mathrm{e}}$ & 8.0 & 10.6 & 70.6 & $76.2^{\mathrm{e}}$ & 5.6 & 7.9 \\
\hline Non-Hodgkin lymphoma & 46.8 & $57.0^{\mathrm{e}}$ & 10.2 & 21.8 & 49.9 & $61.7^{\mathrm{e}}$ & 11.8 & 23.6 \\
\hline Melanoma of the skin & 77.5 & $89.0^{\mathrm{e}}$ & 11.5 & 14.8 & 86.5 & $92.2^{\mathrm{e}}$ & 5.7 & 6.6 \\
\hline Oral cavity and pharynx & 51.8 & $57.4^{\mathrm{e}}$ & 5.6 & 10.8 & 56.1 & $61.5^{\mathrm{e}}$ & 5.4 & 9.6 \\
\hline Leukemia & 34.8 & $47.0^{\mathrm{e}}$ & 12.2 & 35.1 & 37.2 & $45.7^{\mathrm{e}}$ & 8.5 & 22.8 \\
\hline Kidney and renal pelvis & 51.8 & $63.9^{\mathrm{e}}$ & 12.1 & 23.4 & 51.3 & $63.9^{\mathrm{e}}$ & 12.6 & 24.6 \\
\hline Stomach & 15.2 & $22.1^{\mathrm{e}}$ & 6.9 & 45.4 & 17.8 & $25.4^{\mathrm{e}}$ & 7.6 & 42.7 \\
\hline Pancreas & 2.6 & $4.2^{\mathrm{e}}$ & 1.6 & 61.5 & 2.5 & $4.6^{\mathrm{e}}$ & 2.1 & 84.0 \\
\hline Liver and intrahepatic bile duct & 2.2 & $7.7^{\mathrm{e}}$ & 5.5 & 250.0 & 6.4 & $9.6^{\mathrm{e}}$ & 3.2 & 50.0 \\
\hline Brain and other nervous system & 22.8 & $32.7^{\mathrm{e}}$ & 9.9 & 43.4 & 26.0 & $33.4^{\mathrm{e}}$ & 7.4 & 28.5 \\
\hline Esophagus & 4.3 & $14.2^{\mathrm{e}}$ & 9.9 & 230.2 & 6.4 & $14.7^{\mathrm{e}}$ & 8.3 & 129.7 \\
\hline Larynx & 66.4 & 66.7 & 0.3 & 0.5 & 63.5 & 59.6 & -3.9 & -6.1 \\
\hline Breast (female) & - & - & - & - & 74.9 & $87.7^{\mathrm{e}}$ & 12.8 & 17.1 \\
\hline Corpus and uterus, NOS & - & - & - & - & 86.4 & $84.4^{\mathrm{e}}$ & -2.0 & -2.3 \\
\hline Ovary $^{\mathrm{d}}$ & - & - & - & - & 37.6 & $44.0^{\mathrm{e}}$ & 6.4 & 17.0 \\
\hline Cervix uteri & - & - & - & - & 69.0 & $72.7^{\mathrm{e}}$ & 3.7 & 5.4 \\
\hline Thyroid & 91.4 & 93.4 & 2.0 & 2.2 & 93.5 & $97.3^{\mathrm{e}}$ & 3.8 & 4.1 \\
\hline \multicolumn{9}{|l|}{ Age $0-19$ years (childhood cancers) } \\
\hline All sites ${ }^{\mathrm{d}}$ & 57.6 & $77.1^{\mathrm{e}}$ & 19.5 & 33.9 & 68.3 & $81.0^{\mathrm{e}}$ & 12.7 & 18.6 \\
\hline Bone and joint & 43.3 & $71.1^{\mathrm{e}}$ & 27.8 & 64.2 & 56.5 & 63.6 & 7.1 & 12.6 \\
\hline Brain and ONS & 56.8 & $71.8^{\mathrm{e}}$ & 15.0 & 26.4 & 60.2 & $75.3^{\mathrm{e}}$ & 15.1 & 25.1 \\
\hline Hodgkin lymphoma & 85.8 & $96.4^{\mathrm{e}}$ & 10.6 & 12.4 & 88.2 & $95.8^{\mathrm{e}}$ & 7.6 & 8.6 \\
\hline Leukemia & 44.2 & $74.5^{\mathrm{e}}$ & 30.3 & 68.6 & 53.3 & $77.5^{\mathrm{e}}$ & 24.2 & 45.4 \\
\hline ALL & 52.0 & $82.0^{\mathrm{e}}$ & 30.0 & 57.7 & 63.5 & $83.8^{\mathrm{e}}$ & 20.3 & 32.0 \\
\hline AML & 22.8 & $45.5^{\mathrm{e}}$ & 22.7 & 99.6 & 20.5 & $54.2^{\mathrm{e}}$ & 33.7 & 164.4 \\
\hline Neuroblastoma & 51.6 & $65.5^{\mathrm{e}}$ & 13.9 & 26.9 & 56.6 & $65.7^{\mathrm{e}}$ & 9.1 & 16.1 \\
\hline Non-Hodgkin lymphoma & 42.1 & $78.5^{\mathrm{e}}$ & 36.4 & 86.5 & 57.9 & $82.4^{\mathrm{e}}$ & 24.5 & 42.3 \\
\hline Soft tissue & 62.4 & $73.2^{\mathrm{e}}$ & 10.8 & 17.3 & 69.6 & 70.8 & 1.2 & 1.7 \\
\hline Wilms tumor & 72.7 & $92.2^{\mathrm{e}}$ & 19.5 & 26.8 & 76.3 & $91.9^{\mathrm{e}}$ & 15.6 & 20.4 \\
\hline
\end{tabular}

Source: 9 SEER areas (San Francisco-0akland, Connecticut, Detroit-Metropolitan, Hawaii, Iowa, New Mexico, Seattle-Puget Sound, Utah, and Atlanta-Metropolitan),

SEER: Surveillance, Epidemiology, and End Results Program; NOS: not otherwise specified; ONS: other nervous system; ALL: acute lymphocytic leukemia; AML: acute myeloid leukemia.

${ }^{\text {a }}$ Survival rates are based on follow-up of patients through 2001.

${ }^{\mathrm{b}}$ Top 15 cancers includes the top 15 cancers for males and the top 15 cancers for females based on the age-adjusted rate for 1992-2001 for all races combined.

${ }^{\mathrm{c}}$ Absolute change refers to 1995-2000 rate minus 1975-1979 rate whereas proportional change refers to the absolute change divided by the 1975-1979 rate.

${ }^{\mathrm{d}}$ All sites excludes myelodysplastic syndromes and borderline tumors; ovary excludes borderline tumors.

${ }^{\mathrm{e}}$ The difference in rates between $1975-1979$ and $1995-2000$ is statistically significant $(P<0.05)$.

-Survival rate not applicable.

The stage distributions for colorectal, breast, cervical, and prostate cancers are shown by state in Table 7 . The combined stage distributions for the 4 specific cancers among these 29 registries showed lower proportions of early stages and higher proportions of distant and unknown stages (Table 7) than those reported in the SEER Program (Table 6). Furthermore, the stage distributions varied by geographic area for each of the four cancers. The proportion of localized disease ranged from $28.5 \%$ in Nebraska to $44.4 \%$ in Hawaii for colorectal cancer, from $56.5 \%$ in Wyoming to $70.2 \%$ in Hawaii for female breast cancer, from $44.7 \%$ in Arizona to $62.5 \%$ in Utah for cervical cancer, and from $68.1 \%$ in Rhode Island to $95.5 \%$ in Utah for prostate cancer. The proportion of unknown stages of disease also varied by state for each of the four cancers. 
TABLE 6

SEER 5-Year Relative Survival Rates ${ }^{\mathrm{a}}$ and Stage Distribution ${ }^{\mathrm{b}}$ for the Top 15 Cancer Sites, ${ }^{\mathrm{c}}$ 1995-2000

\begin{tabular}{|c|c|c|c|c|c|c|c|c|c|c|}
\hline & \multicolumn{5}{|c|}{ 5-year relative survival rate (\%) } & \multicolumn{5}{|c|}{ Stage distribution } \\
\hline & $\begin{array}{l}\text { All stages } \\
5 \text {-yr rate(\%) }\end{array}$ & $\begin{array}{l}\text { Localized } \\
\text { 5-yr rate(\%) }\end{array}$ & $\begin{array}{l}\text { Regional } \\
5 \text {-yr rate(\%) }\end{array}$ & $\begin{array}{l}\text { Distant } \\
\text { 5-yr rate(\%) }\end{array}$ & $\begin{array}{l}\text { Unstaged } \\
\text { 5-yr rate(\%) }\end{array}$ & $\begin{array}{l}\text { All stages } \\
\text { cases }\end{array}$ & $\begin{array}{l}\text { Localized } \\
\%\end{array}$ & $\begin{array}{l}\text { Regional } \\
\%\end{array}$ & $\begin{array}{l}\text { Distant } \\
\%\end{array}$ & $\begin{array}{l}\text { Unstaged } \\
\%\end{array}$ \\
\hline All sites ${ }^{\mathrm{d}}$ & 64.1 & - & - & - & - & 581,649 & - & - & - & - \\
\hline Prostate & 99.3 & 100.0 & e & 33.5 & 81.3 & 96,225 & 90.2 & e & 5.2 & 4.5 \\
\hline Lung and bronchus & 15.2 & 48.8 & 22.8 & 3.3 & 8.7 & 75,341 & 16.4 & 20.3 & 53.0 & 10.3 \\
\hline Colon and rectum & 63.4 & 90.5 & 67.9 & 9.4 & 35.2 & 63,334 & 37.0 & 39.0 & 18.9 & 5.1 \\
\hline Urinary bladder & 81.7 & 89.7 & 36.9 & 5.5 & 59.0 & 23,885 & 85.2 & 7.9 & 3.3 & 3.5 \\
\hline Non-Hodgkin lymphoma & 59.1 & 71.5 & 63.5 & 47.7 & 66.3 & 24,126 & 32.0 & 13.4 & 45.4 & 9.2 \\
\hline Melanoma of the skin & 90.5 & 95.8 & 52.4 & 16.2 & 76.1 & 21,045 & 87.9 & 4.4 & 3.4 & 4.4 \\
\hline Oral cavity and pharynx & 58.7 & 80.4 & 50.2 & 31.6 & 46.5 & 13,249 & 36.4 & 43.5 & 11.7 & 8.4 \\
\hline Leukemia & 46.4 & - & - & - & - & 15,233 & - & - & - & - \\
\hline Kidney and renal pelvis & 63.9 & 90.9 & 59.7 & 9.5 & 31.6 & 13,092 & 52.4 & 19.8 & 22.0 & 5.8 \\
\hline Stomach & 23.3 & 59.9 & 23.9 & 3.3 & 12.6 & 10,067 & 22.5 & 31.2 & 33.3 & 13.0 \\
\hline Pancreas & 4.4 & 15.2 & 6.3 & 1.6 & 3.8 & 13,212 & 7.5 & 29.3 & 47.2 & 15.9 \\
\hline Liver and intrahepatic bile duct & 8.3 & 18.4 & 6.8 & 4.0 & 3.1 & 6,457 & 29.5 & 11.9 & 35.5 & 23.1 \\
\hline Brain and other nervous system & 33.0 & - & - & - & - & 8,758 & - & - & - & - \\
\hline Esophagus & 14.3 & 29.3 & 13.6 & 3.1 & 11.0 & 5,485 & 26.1 & 28.4 & 27.2 & 18.2 \\
\hline Larynx & 65.1 & 80.0 & 42.3 & 37.5 & 54.5 & 4,937 & 61.7 & 18.3 & 16.1 & 3.9 \\
\hline Thyroid & 96.5 & 99.6 & 95.8 & 68.3 & 87.8 & 9,436 & 65.4 & 23.8 & 7.7 & 3.1 \\
\hline Breast (females) & 87.7 & 97.5 & 79.1 & 20.4 & 56.7 & 91,516 & 63.4 & 29.7 & 4.4 & 2.5 \\
\hline Corpus and uterus, NOS & 84.4 & 95.8 & 67.0 & 22.5 & 56.0 & 17,250 & 72.0 & 16.1 & 7.5 & 4.4 \\
\hline Ovary ${ }^{c}$ & 44.0 & 94.2 & 77.6 & 28.5 & 23.9 & 9,645 & 15.0 & 10.3 & 68.0 & 6.6 \\
\hline Cervix uteri & 72.7 & 92.2 & 55.1 & 17.2 & 59.2 & 6,321 & 55.2 & 30.0 & 8.7 & 6.1 \\
\hline
\end{tabular}

Source: 9 SEER areas (San Francisco-Oakland, Connecticut, Detroit-Metropolitan, Hawaii, Iowa, New Mexico, Seattle-Puget Sound, Utah, and Atlanta-Metropolitan).

SEER: Surveillance, Epidemiology, and End Results Program; NOS: not otherwise specified.

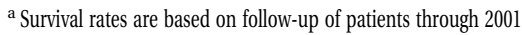

${ }^{\mathrm{b}}$ Cases were staged according to SEER Summary Stage 1977. Cases based on death certificate only and autopsy were excluded.

${ }^{\mathrm{c}}$ Top 15 cancers includes the top 15 cancers for males and the top 15 cancers for females based on the age-adjusted rate for 1992-2001 for all races combined.

${ }^{\mathrm{d}}$ All sites excludes myelodysplastic syndromes and borderline tumors; ovary excludes borderline tumors.

e Localized/regional stages are combined for prostate cases and reported under the "Localized" heading.

-Stage not applicable.

\section{Survival Rates by Race and Ethnicity}

For all cancers combined, cancer-specific survival rates at 5 years after diagnosis ranged from $53.9 \%$ (AI/AN) to $67.6 \%$ (non-Hispanic whites) among men and from $57.0 \%$ (blacks) to $68.7 \%$ (API) among women in the 5 $\mathrm{racial} / \mathrm{ethnic}$ populations studied (Table 8). For both men and women, non-Hispanic white and API patients tended to have higher survival rates than their counterparts for the cancer sites examined, except non-Hispanic white patients, who had the lowest survival rates for cancer of the brain $33.5 \%$ for men and $35.1 \%$ for women) and API patients, who experienced the lowest survival rates for leukemia $39.1 \%$ for men and $38.9 \%$ for women).

Adjusted RRs of cancer death, accounting for age or age and stage, were calculated using non-Hispanic white population as the reference group. Except for myeloma and cancers of the kidney, thyroid, and gallbladder for both sexes, melanoma and other nonepithelial skin cancers for men, and cancer of the liver for women, the overall test for racial/ethnic differences in risk of cancer death was statistically significant for each other cancer site examined and for all cancers combined. The risk of cancer death in every minority group was statistically significantly higher than that of non-Hispanic white patients for all cancers combined, except for API women, who had a similar risk of cancer death as their nonHispanic white counterparts. Black men experienced $9-67 \%$ higher adjusted risks of dying of the index cancer than non-Hispanic white men for 12 of 13 individual cancer sites with statistically significant racial/ethnic differences; black women experienced $7-82 \%$ higher adjusted risks for 12 of 14 such individual cancers, except for cancers of the brain (both sexes) and stomach (women), which were lower. Hispanic white and AI/AN patients generally had higher risks of dying from the index cancer than their non-Hispanic white and API counterparts. Both API men and women experienced the lowest RRs for each of the sex-specific major cancers (prostate, female breast, 


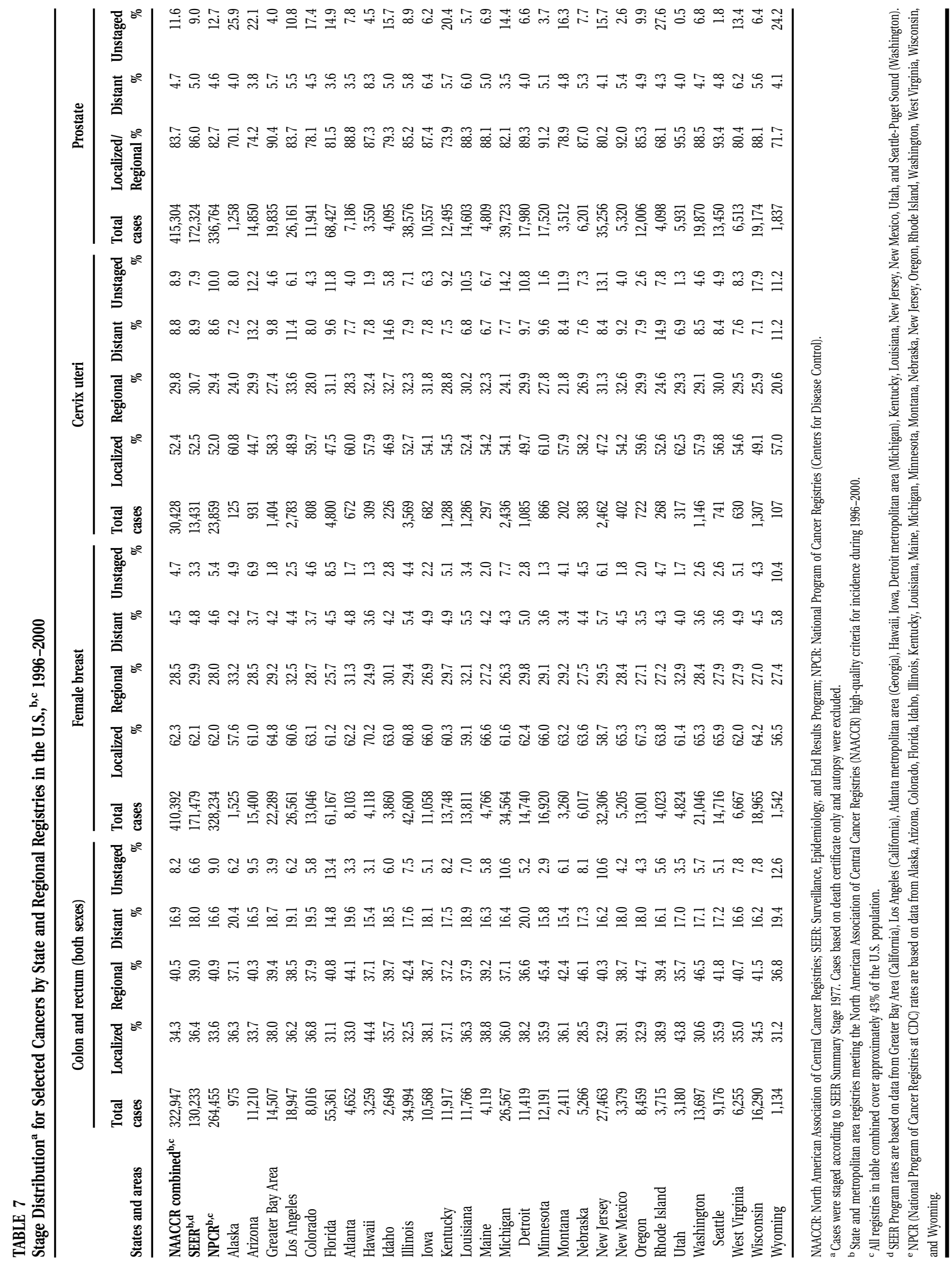


TABLE 8

SEER 5-Year Cancer-Specific Survival and Stage-Adjusted and Age-Adjusted Relative Risk of Cancer Death by Cancer Type, Race/Ethnicity, and Sex, 1992-2000

\begin{tabular}{|c|c|c|c|c|c|c|c|c|c|c|}
\hline & \multicolumn{5}{|c|}{ Cause-specific survival (\%) } & \multicolumn{5}{|c|}{ Adjusted relative risk $(95 \% \mathrm{CI})$ of cancer deaths $\mathrm{s}^{\mathrm{a}}$} \\
\hline & $\begin{array}{l}\mathrm{NH} \\
\text { whites }\end{array}$ & $\begin{array}{l}\mathrm{H} \\
\text { whites }\end{array}$ & Blacks & API & $\mathrm{AI} / \mathrm{AN}$ & $\begin{array}{l}\mathrm{NH} \\
\text { whites }\end{array}$ & H whites & Blacks & API & AI/AN \\
\hline Male & & & & & & (Ref.) & & & & \\
\hline All sites & 67.6 & 65.1 & 62.1 & 60.5 & 53.9 & 1.0 & $1.16(1.14-1.18)$ & $1.26(1.24-1.28)$ & $1.26(1.23-1.28)$ & $1.69(1.59-1.79)$ \\
\hline Brain and other nervous system & 33.5 & 46.0 & 45.2 & 42.6 & 59.7 & 1.0 & $0.97(0.88-1.07)$ & $0.98(0.87-1.10)$ & $0.79(0.68-0.92)$ & $0.77(0.48-1.24)$ \\
\hline Colon and rectum & 64.0 & 60.9 & 56.1 & 66.7 & 62.3 & 1.0 & $1.05(0.99-1.11)$ & $1.26(1.20-1.32)$ & $0.95(0.90-1.00)$ & $1.14(0.95-1.35)$ \\
\hline Esophagus & 18.9 & 20.6 & 13.4 & 17.4 & 18.6 & 1.0 & $1.05(0.94-1.17)$ & $1.29(1.19-1.40)$ & $1.14(1.01-1.28)$ & $1.13(0.80-1.60)$ \\
\hline Gallbladder & 29.1 & 26.9 & 39.2 & 36.2 & 38.4 & 1.0 & $1.20(0.91-1.60)$ & $1.18(0.81-1.74)$ & $1.24(0.90-1.72)$ & $1.26(0.66-2.41)$ \\
\hline Kidney and renal pelvis & 66.1 & 65.4 & 69.7 & 62.6 & 70.2 & 1.0 & $1.03(0.94-1.14)$ & $1.06(0.95-1.18)$ & $0.96(0.84-1.09)$ & $1.14(0.83-1.58)$ \\
\hline Larynx & 81.1 & 75.1 & 72.6 & 81.8 & $63.8^{\mathrm{b}}$ & 1.0 & $1.37(1.13-1.66)$ & $1.44(1.25-1.65)$ & $0.88(0.65-1.20)$ & $2.56^{\mathrm{b}}(1.21-5.40)$ \\
\hline Leukemia & 53.3 & 53.0 & 46.2 & 39.1 & 45.7 & 1.0 & $1.37(1.27-1.48)$ & $1.40(1.28-1.52)$ & $1.71(1.56-1.89)$ & $1.43(1.03-1.99)$ \\
\hline Liver and intrahepatic bile duct & 12.7 & 15.1 & 7.8 & 15.6 & 20.1 & 1.0 & $1.09(1.01-1.18)$ & $1.20(1.10-1.31)$ & $0.91(0.85-0.98)$ & $1.08(0.83-1.39)$ \\
\hline Lung and bronchus & 16.0 & 14.4 & 13.7 & 17.3 & 14.0 & 1.0 & $1.08(1.04-1.12)$ & $1.09(1.06-1.11)$ & $0.90(0.87-0.93)$ & $1.23(1.10-1.36)$ \\
\hline Melanoma of the skin & 86.5 & 77.1 & 77.7 & 76.5 & 80.1 & 1.0 & $1.08(0.88-1.34)$ & $0.88(0.55-1.41)$ & $1.11(0.75-1.64)$ & $1.29(0.53-3.12)$ \\
\hline Myeloma & 36.2 & 39.7 & 40.4 & 46.6 & 33.1 & 1.0 & $1.00(0.89-1.13)$ & $1.01(0.92-1.11)$ & $0.80(0.68-0.94)$ & $1.25(0.80-1.94)$ \\
\hline Non-Hodgkin lymphoma & 63.2 & 61.6 & 65.0 & 55.5 & 56.1 & 1.0 & $1.32(1.22-1.42)$ & $1.21(1.10-1.32)$ & 1.32 (1.21-1.45) & $1.57(1.12-2.21)$ \\
\hline Oral cavity and pharynx & 77.5 & 73.2 & 63.3 & 71.2 & 60.7 & 1.0 & $1.25(1.09-1.42)$ & $1.67(1.52-1.83)$ & $1.22(1.07-1.38)$ & $1.56(1.15-2.12)$ \\
\hline Other nonepithelial skin ${ }^{\mathrm{d}}$ & 97.8 & 99.4 & 98.2 & 98.6 & 100.0 & 1.0 & $0.55(0.22-1.38)$ & $1.28(0.63-2.63)$ & $0.92(0.29-2.97)$ & $\mathrm{c}$ \\
\hline Pancreas & 5.6 & 7.5 & 5.5 & 7.6 & 9.6 & 1.0 & $1.08(1.00-1.16)$ & $1.15(1.08-1.22)$ & $0.97(0.90-1.05)$ & $1.07(0.82-1.41)$ \\
\hline Prostate & 92.0 & 90.0 & 88.3 & 92.3 & 83.0 & 1.0 & $1.12(1.05-1.19)$ & $1.31(1.25-1.36)$ & $0.70(0.65-0.76)$ & $1.81(1.46-2.24)$ \\
\hline Stomach & 34.9 & 28.0 & 27.3 & 32.7 & 18.8 & 1.0 & $1.26(1.18-1.36)$ & $1.33(1.24-1.43)$ & $1.10(1.02-1.18)$ & $1.87(1.52-2.30)$ \\
\hline Thyroid & 92.3 & 92.8 & 91.5 & 94.3 & 96.0 & 1.0 & $0.99(0.68-1.44)$ & $1.15(0.69-1.90)$ & $0.79(0.51-1.22)$ & $0.87(0.28-2.75)$ \\
\hline Urinary bladder & 85.1 & 84.0 & 75.5 & 84.3 & 75.5 & 1.0 & $1.09(0.95-1.26)$ & $1.43(1.27-1.61)$ & $0.86(0.73-1.01)$ & $1.14(0.70-1.87)$ \\
\hline \multicolumn{11}{|l|}{ Female } \\
\hline All sites & 67.0 & 66.8 & 57.0 & 68.7 & 60.4 & 1.0 & $1.20(1.18-1.22)$ & $1.52(1.50-1.55)$ & $1.01(0.99-1.03)$ & $1.54(1.45-1.64)$ \\
\hline Brain and other nervous system & 35.1 & 46.2 & 43.9 & 45.4 & 49.2 & 1.0 & $1.05(0.95-1.17)$ & $0.88(0.78-1.00)$ & $0.75(0.63-0.89)$ & $0.78(0.44-1.38)$ \\
\hline Breast & 87.5 & 83.0 & 75.0 & 89.4 & 79.6 & 1.0 & $1.22(1.16-1.28)$ & $1.75(1.68-1.82)$ & $0.90(0.85-0.97)$ & $1.55(1.32-1.81)$ \\
\hline Cervix uteri & 77.2 & 81.1 & 69.5 & 78.0 & 75.8 & 1.0 & $0.77(0.69-0.85)$ & $1.21(1.09-1.34)$ & $0.83(0.71-0.96)$ & $0.97(0.64-1.47)$ \\
\hline Colon and rectum & 63.4 & 61.3 & 57.0 & 68.2 & 58.2 & 1.0 & $1.05(0.99-1.11)$ & $1.18(1.13-1.23)$ & $0.90(0.85-0.96)$ & $1.38(1.16-1.64)$ \\
\hline Corpus and uterus & 86.6 & 85.4 & 69.9 & 87.4 & 83.2 & 1.0 & $1.22(1.09-1.38)$ & $1.82(1.66-1.99)$ & $1.08(0.94-1.24)$ & 1.69 (1.09-2.63) \\
\hline Gallbladder & 25.6 & 32.3 & 30.2 & 27.7 & 32.3 & 1.0 & $1.05(0.91-1.21)$ & $0.91(0.73-1.14)$ & $0.89(0.70-1.13)$ & $0.82(0.52-1.28)$ \\
\hline Kidney and renal pelvis & 66.4 & 69.6 & 70.1 & 68.4 & 64.2 & 1.0 & $0.94(0.83-1.07)$ & $0.99(0.87-1.12)$ & $0.78(0.64-0.96)$ & $1.16(0.83-1.62)$ \\
\hline Leukemia & 51.7 & 53.0 & 43.9 & 38.9 & 46.5 & 1.0 & $1.33(1.21-1.45)$ & $1.36(1.24-1.49)$ & $1.75(1.57-1.96)$ & $1.48(1.07-2.06)$ \\
\hline Liver and intrahepatic bile duct & 15.9 & 18.5 & 11.8 & 14.8 & 25.0 & 1.0 & $1.07(0.96-1.20)$ & $1.14(1.00-1.30)$ & $0.99(0.89-1.11)$ & $0.94(0.66-1.33)$ \\
\hline Lung and bronchus & 20.2 & 18.1 & 18.1 & 19.8 & 17.2 & 1.0 & $1.04(1.00-1.09)$ & $1.07(1.04-1.11)$ & $0.89(0.85-0.93)$ & $1.22(1.06-1.40)$ \\
\hline Melanoma of the skin & 92.2 & 86.8 & 71.5 & 78.8 & 80.1 & 1.0 & $1.23(0.97-1.55)$ & $1.82(1.20-2.77)$ & $1.43(0.89-2.32)$ & $0.98(0.41-2.39)$ \\
\hline Myeloma & 32.2 & 31.8 & 35.2 & 40.5 & 23.4 & 1.0 & $1.08(0.96-1.23)$ & $1.00(0.92-1.10)$ & $0.84(0.70-1.00)$ & $1.43(0.93-2.20)$ \\
\hline Non-Hodgkin lymphoma & 62.9 & 62.1 & 64.0 & 64.2 & 63.0 & 1.0 & $1.21(1.11-1.31)$ & $1.29(1.17-1.42)$ & $1.09(0.98-1.21)$ & $1.42(0.99-2.05)$ \\
\hline Oral cavity and pharynx & 75.8 & 76.3 & 70.6 & 75.9 & 69.0 & 1.0 & $1.04(0.86-1.27)$ & $1.32(1.14-1.54)$ & $1.02(0.84-1.23)$ & $1.29(0.73-2.28)$ \\
\hline Ovary & 43.6 & 53.2 & 46.3 & 57.4 & 45.5 & 1.0 & $1.01(0.94-1.08)$ & $1.19(1.10-1.29)$ & $0.96(0.87-1.07)$ & $1.10(0.84-1.43)$ \\
\hline Pancreas & 5.0 & 8.9 & 5.4 & 8.4 & $\mathrm{c}$ & 1.0 & $1.01(0.94-1.08)$ & $1.09(1.03-1.15)$ & $0.87(0.80-0.94)$ & $1.14(0.89-1.47)$ \\
\hline Stomach & 31.0 & 29.0 & 32.7 & 33.0 & 21.6 & 1.0 & $1.12(1.03-1.22)$ & $0.96(0.88-1.05)$ & $0.98(0.90-1.07)$ & 1.53 (1.19-1.97) \\
\hline Thyroid & 96.5 & 95.6 & 96.3 & 94.9 & 98.1 & 1.0 & $1.22(0.95-1.58)$ & $0.94(0.64-1.37)$ & $1.24(0.95-1.63)$ & $1.12(0.28-4.54)$ \\
\hline Urinary bladder & 79.1 & 75.5 & 62.9 & 76.1 & $76.7^{\mathrm{b}}$ & 1.0 & $1.12(0.93-1.34)$ & $1.38(1.20-1.58)$ & $0.98(0.79-1.23)$ & $1.79^{\mathrm{b}}(0.67-4.77)$ \\
\hline
\end{tabular}

Source: 12 SEER areas (San Francisco-Oakland, Connecticut, Detroit-Metropolitan, Hawaii, Iowa, New Mexico, Seattle-Puget Sound, Utah, Atlanta-Metropolitan, San Jose-Monterey, Los Angeles, and AI/AN in Alaska).

SEER: Surveillance, Epidemiology, and End Results Program of the National Cancer Institute, NH whites: non-Hispanic whites; H whites: Hispanic whites; API: Asian/Pacific Islander; AI/AN: American Indian/Alaska Native; 95\% CI: 95\% confidence interval.

${ }^{a}$ Up to 5 years of follow-up. Using stratified Cox models, the relative risks for all cancers combined are adjusted for age at diagnosis, and those for individual cancer sites are adjusted for age and tumor stage. $P$ values are $<0.05$ for the overall test of racial/ethnic differences in the relative risks of cancer deaths using stratified Cox models by cancer site and sex; except for Kidney and renal pelvis, Thyroid, Myeloma, and Gallbladder for both sexes, for Liver and Intrahepatic bile duct for female, and for Melanoma of the skin and Other nonepithelial skin for male (all have $P$ values $>0.06$ and are marked with gray background). ${ }^{\mathrm{b}}$ Cohort size $<25$ (24 for male AI/AN Larynx and 18 for female AI/AN Urinary bladder).

${ }^{\mathrm{c}}$ Statistic could not be calculated for female AI/AN diagnosed with cancer of pancreas because the last patient was censored before the end of 5-year follow-up (censored at 35 months) and for male AI/AN diagnosed with other nonepithelial skin because none of the 39 patients died of Other nonepithelial skin cancer at the end of 5-year follow-up.

${ }^{d}$ For male cancer of other nonepithelial skin, nearly $70 \%$ were Kaposi sarcoma and, of those who died, $80 \%$ had acquired immunodeficiency syndrome (AIDS) as the underlying cause of death. These were not considered cancer deaths. 
lung, and colon/rectum), with RRs ranging from 0.70-0.95. In addition, API patients had the lowest RRs for cancers of the brain (women), liver (men), and pancreas (both sexes). However, non-Hispanic white men and women experienced the highest risks of brain cancer death whereas API men and women had the highest risks of leukemia death.

\section{DISCUSSION}

\section{Overall Cancer Incidence, Mortality, and Survival Trends}

The current study data show considerable progress in reducing the cancer burden in the U.S. Overall cancer death rates have continued to decrease since the early 1990s in both men and women and for many of the top 15 cancer sites, including lung, colon/rectum, and prostate in men and colon/rectum and breast in women. Overall incidence rates also have decreased, although they stabilized when adjusted for a delay in reporting. Survival rates have increased for many cancers over the last 20 years.

The decrease in overall cancer death rates represents a change from last year's annual report, when the declines were reported to have leveled off in the most recent time period in women and both sexes combined. ${ }^{6}$ With the additional data for 2001, it appears that the declines in mortality rates that began in the early 1990s are continuing, supporting an earlier interpretation that the apparent change in trend may be a consequence of changes in coding rules for underlying cause of death in the ICD-10, which were implemented with 1999 mortality data and revised population. $^{25}$

Changes in incidence may result from changes in the prevalence of risk factors and/or changes in detection practices due to the introduction or increased use of screening/diagnostic techniques. Furthermore, incidence trends can be affected by reporting delay. The overall trend in incidence for both sexes combined appears to have been heavily influenced by reporting delay and rapidly changing prostate cancer trends; prostate cancer decreased quickly between 1992-1995, after a period of rapid increase. When prostate cancer incidence rates were excluded from the trend analysis, the incidence rates for both sexes combined were stable during 1987-2001. Overall incidence rates also became stable between 1995-2001 when adjusted for reporting delay.

The gains in survival rates for all cancer sites combined and for the most common cancers over the last 20 years, which have been accompanied by decreases in death rates, may reflect improved treatment, earlier detection of cancers with effective treatments, and improved supportive and general medical care. However, the introduction of or improvements in early detection or diagnostic tests may increase observed survival rates spuriously by advancing the time of diagnosis of disease without prolonging life (lead-time bias), by the preferential detection of slower growing tumors (length-time bias), or by the detection of indolent cases that never would have been diagnosed in the absence of such diagnostic techniques (overdiagnosis bias). ${ }^{9,26,27}$ Although examining temporal changes in disease stage at diagnosis and stage-specific survival may be helpful in understanding temporal trends in survival, they must be interpreted with caution because changes in stage-specific survival, especially for localized stage disease, also can be affected by lead-time, length-time, and overdiagnosis

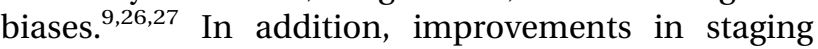
techniques or changes in staging systems can influence stage-specific survival. ${ }^{28}$ Assessing the relative contribution of changes in disease detection, classification, and treatment is difficult when explaining temporal survival changes.

Survival rates for all cancers combined also may change over time or differ across racial and ethnic populations because of changes or differences in the mix of cancers. For example, the absolute change in survival for cancers of all sites in the last 20 years was substantially greater for men than for women. Much of the increase in survival for men reflects the increasing incidence and survival rate for prostate cancer during the period. When prostate cancer cases were excluded, the all cancers combined relative survival rate for men was $37.5 \%$ between $1975-1979$ and $48.1 \%$ between 1995-2000, an absolute gain of $10 \%$ compared with approximately $20 \%$ with the inclusion of prostate cancer. The lower survival rate for men compared with women in the recent period, after the exclusion of prostate cancer, primarily results from a different mix of cancers, although women have a slight survival advantage for a number of cancers. The survival rate for a specific cancer also may be affected if there is variation by histologic type and the distribution of histologic types changes over time.

Differences in survival between racial and ethnic populations may be influenced by stage at diagnosis, the measurement biases discussed earlier, the prevalence of comorbidities, and the quality of cancer treatment and supportive care. Socioeconomic differences undoubtedly play a role; members of most racial and ethnic minorities are more likely to be poor, to have a lower level of income, and to lack health insurance compared with non-Hispanic white populations. ${ }^{29}$ Historically, the prevalence of screening for breast, colorectal, and cervical cancers has been lower for members of racial and ethnic minorities compared with non-Hispanic white populations. ${ }^{30}$ As a result, 
minority populations are more likely to be diagnosed at a more advanced stage of disease, when treatment is either not recommended or is less effective.

Differences in cause-specific survival rates by race and ethnicity may be influenced by differences in stage and age distribution; however, analyses of the RR of cancer death controlled for these factors. Even with control for stage, residual effects of screening, such as differences in the extent of disease within stage and lead-time, length-time, and overdiagnosis biases, may play a role. Other possible explanations for differential survival after a cancer diagnosis include socioeconomic barriers to timely and high-quality cancer care, cultural barriers and beliefs that may influence the treatment decision, and the presence of comorbidities. ${ }^{29,31-33}$ For many cancers, there are limited or no data available to explain survival differences, with much of the available data pertaining only to white and black patients. The limited data available frequently document differences in the receipt of optimal treatment based on socioeconomic status and race/ethnicity, with little evidence that response to treatment varies among racial and ethnic groups when accounting for stage of disease and other important prognostic variables. ${ }^{33}$

The following sections describe possible reasons for temporal trends and variations in the incidence, mortality, and survival by race and ethnicity for the 4 common cancers and for cancers with an at least $10 \%$ absolute gain in the survival rate over the past 20 years in either men or women.

\section{Prostate Cancer}

The recent increase in prostate cancer incidence most likely reflects a return to baseline trends after a period of rapidly increasing, then decreasing, rates due to the introduction of the prostate-specific antigen (PSA) test. ${ }^{34}$ Both PSA-related early detection and improved treatments may have contributed to the remarkable decreases in prostate cancer mortality noted since the early 1990s. ${ }^{6}$ Reasons for the disproportionately higher prostate cancer incidence and death rates in black men compared with other racial ethnic populations are unknown. Age, race, and family history are the only well-documented risk factors. ${ }^{35}$ Although one recent study linked agricultural chemical exposure to prostate cancer, ${ }^{36}$ population-attributable risk differences in chemical exposure are unlikely to explain the large differences in prostate cancer incidence and death rates observed between black and white men.

The 5-year relative survival rate for prostate cancer increased significantly from $70 \%$ in the mid-1970s to $99 \%$ between 1995-2000, the largest absolute increase $(29 \%)$ reported for any cancer. A shift in the detection of new prostate cancer cases from distantstage to early-stage disease through the widespread use of PSA testing has been reported ${ }^{37}$ and most likely contributed to the improved survival. However, because a high proportion of men are found to have clinically occult cancer at autopsy, ${ }^{38,39}$ it is assumed that some prostate cancers identified by PSA would not have otherwise presented as clinical disease. ${ }^{40}$ The extent to which overdiagnosis, length-time, and leadtime biases have contributed to improvements in survival is unknown, but believed to be substantial.

Improved survival from prostate cancer also may be due to the dissemination of hormonal therapy for early-stage and advanced stage disease. ${ }^{41,42}$ A recent pooled analysis of prostate cancer randomized trials found that the 10-year survival rate was significantly higher in men treated immediately with hormonal therapy $(74 \%)$ compared with those who deferred such treatment $(62 \%) .^{43}$ Substantial advances in radiation therapy techniques in the past decade also may have contributed to gains in survival. ${ }^{44}$ The role of radical prostatectomy for localized disease has yet to be clarified.

After controlling for age and stage of diagnosis, the RR of cancer death once prostate cancer is diagnosed is highest for AI/AN men, followed by black and Hispanic white men; API men have a significantly lower risk than non-Hispanic white men. Low socioeconomic status, lack of health insurance, and low literacy rates can delay diagnosis and reduce access to optimal therapies. ${ }^{45}$ Treatment differences such as a higher rate of radical prostatectomy for early-stage prostate cancer in white patients compared with black patients have been documented ${ }^{46}$ however, the impact on survival differences is unknown.

\section{Lung Cancer}

Changing temporal patterns in the occurrence of lung cancer are closely tied to historic cigarette smoking patterns, with the regular uptake of smoking and subsequent smoking reductions occurring later in women compared with men. ${ }^{47}$ Among men, lung cancer incidence and mortality have been declining since the early 1980s and 1990s, respectively. In women, incidence rates have declined since 1998 and mortality rates have stabilized since 1995 for the first time, after increasing for several decades. This first-time decrease in the incidence rates in women in the SEER areas appears to be an early indication of the national trend because reductions in cigarette consumption started earlier in some areas covered by SEER compared with the other parts of the U.S. ${ }^{48}$

Progress in the treatment of lung cancer has been extremely limited. ${ }^{49,50}$ Greater than $50 \%$ of cases are 
diagnosed at a distant stage, for which the 5-year relative survival is only $3.3 \%$ (Table 6 ). Surgical resection may be curative for early-stage nonsmall cell lung cancer (NSCLC), with recent clinical studies showing improved survival with neoadjuvant chemotherapy. ${ }^{49}$ With the exception of API patients, the RR of dying from lung cancer after diagnosis was $4-23 \%$ higher in minority populations compared with non-Hispanic white patients. Differences in survival between black and white patients with NSCLC are largely explained by treatment differences, with $64.0 \%$ of black patients who are diagnosed at an early stage undergoing surgery compared with $76.7 \%$ of whites ${ }^{51}$ and $28 \%$ of black patients diagnosed with metastatic disease receiving chemotherapy after seeing an oncologist compared with $35 \%$ of white patients. ${ }^{52}$ In addition to ensuring equal access to high-quality treatment, improved survival from lung cancer may be achieved by earlier detection. Important research efforts include the National Lung Screening Trial, which has enrolled nearly 50,000 current or former smokers in a study to compare whether screening with spiral computed tomography or chest X-rays is better at reducing deaths from lung cancer (available from URL: http://www. nci.nih.gov/nlst).

\section{Colorectal Cancer}

Decreases in colorectal cancer incidence and mortality rates have been largely attributed to the detection and removal of precancerous polyps, the early detection of tumors through screening, and improved treatments. $^{4,6}$ They also may reflect the increased use of hormone replacement therapy in women and antiinflammatory drugs, both of which appear to reduce the risk of colon cancer. ${ }^{53,54}$ A recent publication found a decreased risk of colorectal cancer but a more advanced stage of disease (increased lymph node and metastatic disease) among women receiving estrogen and progestin regimens. ${ }^{55}$ The elevated incidence and death rates in the black population may reflect cultural and socioeconomic differences in physical inactivity, dietary habits, use of tobacco, and access to and utilization of preventive services. ${ }^{56}$

From the mid-1970s until 1995-2000, the 5-year relative survival rate increased from $52 \%$ to $63 \%$ in women and from $50 \%$ to $64 \%$ in men, an increase that was second only to prostate cancer. The rate of colorectal screening remains low nationally $(<45 \%$ based on the 2000 National Health Interview survey ${ }^{30}$ ) and the potential benefit with broader utilization has yet to be achieved. However, the use of colorectal screening is higher in non-Hispanic white men and women than in other racial and ethnic populations.

A significant advance in colorectal cancer treat- ment was the introduction of 5-fluoroucil-based adjuvant chemotherapy for patients with surgically resectable Stage III colon cancer in the late 1980s, which reduced mortality by as much as $30 \%{ }^{57,58}$ Lower rates of adjuvant therapy among black patients ${ }^{59-62}$ may contribute to differences in cancer survival. Other studies have documented disparities between black and non-Hispanic white patients with regard to the receipt of surgical and radiation treatments for colorectal cancer. ${ }^{62,63}$

\section{Female Breast Cancer}

Recent increases in female breast cancer incidence reflect the increased use of mammography, ${ }^{6,64}$ and perhaps an increased prevalence of obesity and the use of hormone replacement therapy. ${ }^{64}$ The steady decline in female breast cancer mortality since 1990 has been attributed, in part, to early detection ${ }^{6}$ and the increased use of hormonal and adjuvant chemotherapies $^{65,66}$ and the resulting improved survival, which increased by $13 \%$ since the mid-1970s.

Mammography screening increased in all segments of the U.S. population, although rates among minority populations lagged behind rates in white women. ${ }^{30}$ White and API women are more likely to be diagnosed at a localized stage than women of other racial and ethnic populations. ${ }^{67}$ Even when controlled for age and stage at diagnosis, black, Hispanic white, and AI/AN women had an increased RR of mortality after diagnosis compared with white and API women. Reasons for differential breast cancer survival between white and black women have been studied extensively. Several studies documented treatment differences between black and white women, ${ }^{68-70}$ and others found treatment and survival differences between black and white patients diminished when controlling for socioeconomic factors, such as lack of insurance coverage. ${ }^{71,72}$ Tumor and clinical characteristics such as estrogen receptor status, tumor size, tumor stage at diagnosis, and neutropenia also may influence the types and courses of treatment and survival, ${ }^{64,73-76}$ although the differential availability of more biologically targeted treatment options also may contribute to variations in outcome.

\section{Non-Hodgkin Lymphoma}

NHL incidence trends partly reflect an increase in acquired immunodeficiency syndrome-associated NHL cases that began in the early 1980s and decreased in the 1990s. ${ }^{77}$ The increased incidence in white compared with black populations in the U.S. precedes the rise in human immunodeficiency virus (HIV)-related NHL, and has remained remarkably consistent over time. ${ }^{18}$ Recent declines in NHL mortality may reflect 
decreases in HIV-associated NHL incidence $\operatorname{rates}^{77}$ and improved treatments. ${ }^{78}$

NHL comprises a range of pathologic types with varying prognoses. ${ }^{79,80}$ Survival trends are influenced by the increase in HIV-related NHL cases, which have a poorer prognosis. ${ }^{80}$ Nonetheless, modest gains in overall survival have been observed. Improved treatments for indolent NHL include radiotherapy for early-stage disease $\mathrm{e}^{81,82}$ and single-agent and multiagent chemotherapy for advanced-stage disease. ${ }^{83-85}$ For large cell lymphoma, an aggressive NHL, multiagent chemotherapy was shown in the 1970s and 1980 s to achieve disease remission and long-term, disease-free survival in $35 \%$ of patients ${ }^{86,87}$ More recently, rituximab, a monoclonal antibody directed against the CD20 antigen, was found to have activity against both indolent and aggressive $\mathrm{NHL}^{88}$ and autologous bone marrow transplantation was found to increase survival among patients with recurrent, aggressive NHL. ${ }^{89,90}$ Although all racial and ethnic minority groups have a higher RR of cancer death from NHL once diagnosed compared with non-Hispanic whites, no published study to date has assessed prognostic or treatment variations that may underlie these differences.

\section{Melanoma}

The continued increase in melanoma incidence rates may reflect increased recreational sun exposure as well as early detection, resulting mainly from increased awareness by health providers and the general public. ${ }^{91}$ The markedly higher incidence and mortality for white patients reflects their enhanced susceptibility to the harmful effects of sun exposure..$^{92}$ The absolute gain in melanoma survival in males $(12 \%)$ and females $(6 \%)$ may be related both to early detection and improved treatment. Proportions and rates of melanomas diagnosed at a localized stage and as thin lesions have increased in the U.S. ${ }^{91,93}$ Surgical resection, the major curative therapy for melanoma at the current time, has undergone significant improvements as a result of documenting optimal margins based on the depth of the primary lesion. ${ }^{94}$ The benefits of immunotherapy (interferon and interleukin-2) in recurrence-free survival and overall survival have been demonstrated in clinical trials, ${ }^{95}$ and the use of recombinant interleukin-2 for patients with metastatic melanoma recently was approved. ${ }^{96}$

\section{Leukemia}

The incidence of leukemia was stable for both males and females between 1975-1995, when rates began to decline for males only. Leukemia is more common in white populations than in other racial and ethnic groups, paralleling international patterns of increased incidence in Western and Northern Europe, North America, and Australia. Long-term decreases in leukemia death rates reflect dramatic improvements in survival from childhood leukemia, as well as modest improvements in survival for some leukemia subtypes in adults. Among children and adults combined, leukemia survival rose from $35.9 \%$ in $1975-1979$ to $46.4 \%$ in 1995-2000, whereas for leukemia patients diagnosed at age $\geq 20$ years, relative survival rates improved from $34 \%$ to $42 \% .{ }^{18}$ As a result of multiagent chemotherapy and central nervous system prophylaxis, relative survival for adult patients with acute lymphocyte leukemia (ALL) has improved from $11 \%$ in 1975-1979 to $28 \%$ in $1995-2000,{ }^{18,97,98}$ whereas survival among adults with chronic lymphocyte leukemia improved only slightly from the 1970s to the 1990 s $(69 \%$ vs. 73\%). ${ }^{18}$ Acute myeloid leukemia remains a highly fatal malignancy, although the 5 -year relative survival improved from $6 \%$ to $17 \%$ over the last 20 years. Combined chemotherapy with daunorubicin and cytarabine remains the treatment of choice for most patients, ${ }^{99}$ and improvements in survival may be attributed in part to improvements in supportive care, including the treatment of infections. ${ }^{99}$ The 5-year relative survival for patients with chronic myelocytic leukemia (CML) also has increased. Historic treatment advances include chemotherapy with hydroxyurea and busulfan, and chemotherapy combined with interferon- $\alpha .{ }^{100}$ More recent advances include allogeneic bone marrow transplant and treatment with imatinib mesylate, which has been demonstrated to be highly effective in inducing and sustaining disease remission when used as a first course of treatment. ${ }^{101,102}$ Survival improvements resulting from imatinib mesylate are too recent to be reflected in 1995-2000 statistics.

The RR of death among leukemia patients is markedly higher for all racial and ethnic minority populations compared with non-Hispanic white populations. The lowest survival rates observed in the API leukemia patients might be attributable to the $62 \%$ of API leukemia patients in SEER areas who were diagnosed with the more lethal subtypes of myeloid and monocytic leukemia compared with $44-50 \%$ of other racial/ethnic populations. Treatment differences that may contribute to differential survival by race and ethnicity have not been studied. In the nine SEER areas, black, Hispanic, and AI/AN children with ALL have poorer survival than white and API children; ${ }^{103}$ other reports have demonstrated that black children with ALL are more likely to present with unfavorable prognostic features. ${ }^{104}$ However, in a single pediatric cancer hospital in which all patients received the same 
treatment without regard to ability to pay, black and white children had the same rate of survival and cure. ${ }^{105}$

\section{Kidney Cancer}

Reasons for the increasing incidence of kidney cancer are not clear, ${ }^{106}$ but may reflect newer diagnostic techniques ${ }^{107,108}$ and an increased prevalence of obesity, ${ }^{109,110}$ which is a known risk factor for kidney cancer. ${ }^{111}$ Despite the rising incidence, mortality rates have been stable since 1991 for men and since 1992 for women. Black men and women have the highest incidence rates among all racial and ethnic populations, but mortality rates are approximately equal for white and black populations and highest for AI/AN patients. Reasons for increased mortality among the AI/AN population are unknown. There is considerable variation in kidney cancer mortality in AI/AN persons by region, with death rates being particularly high in the Northern plains. ${ }^{112}$ Approximately $50 \%$ of cancers of the kidney are diagnosed at the localized stage, for which the 5 -year relative survival is $>90 \%$ (Table 6). Surgery remains the primary treatment, with the benefit of adjuvant chemotherapy and radiotherapy considered to be unproven. ${ }^{113}$ Improved survival may result in part from lowered surgical mortality rates due to advances in anesthesia and presurgical and postsurgical management. ${ }^{113}$ When adjusted for age and stage of disease, no significant differences in the RRs of cancer death once diagnosed were noted between racial and ethnic groups.

\section{Childhood Cancers}

Since the introduction of chemotherapy for the treatment of childhood leukemia in the 1940s, the prognosis for childhood cancers has improved remarkably, from almost uniformly fatal before the mid-1960s to a $55 \%$ survival rate in the mid-1970s, to $>75 \%$ survival in the late 1990s. This improvement has been attributed to the success of chemotherapy for childhood ALL and the incorporation of chemotherapy into treatment regimens that previously relied on surgery or radiotherapy for common forms of childhood cancers such as Wilms' tumor, lymphoma, and osteosarcoma. ${ }^{114}$ Increased survival from childhood cancers also may be correlated in part with improved quality of care through the pediatric cancer cooperative groups. Currently $70 \%$ of pediatric cancer patients age $<15$ years receive their care in pediatric cancer treatment centers. ${ }^{115}$

Although the 5-year survival has improved remarkably for most childhood cancers over the past two decades, with nearly all children with Hodgkin lymphoma surviving their disease, the survival rate is still low $(<50 \%)$ for some childhood cancers such as acute myeloid leukemia, reflecting a need for an improved understanding of the mechanism of resistance to therapy. ${ }^{116}$

Age-specific analyses demonstrated that survival rates did not vary by age for all sites combined, but some variations were evident for individual sites. For example, the survival rates for ALL were $91 \%$ for patients ages 1-4 years but only approximately $60 \%$ for infants and patients ages 15-19 years. This may in part be related to the lower enrollment rates of adolescents and young adults in clinical trails compared with that of children. ${ }^{117}$ In general, survival rates improved more for boys than girls, removing much of the advantage in girls' overall survival noted between 19751979.

\section{Variations in Disease Stage at Diagnosis by Cancer Registry}

Geographic variation in the stage of disease at diagnosis was most evident for cancers of the colon/rectum, cervix, and prostate, whereas much less variation was noted for female breast cancer. The percentages in Table 7 are not adjusted for age, which may influence stage distribution. Other differences in cancer stage at diagnosis may be related to differences in the population composition by race, ethnicity, ${ }^{118,119}$ socioeconomic status, ${ }^{120-123}$ and health insurance status, ${ }^{124,125}$ all of which can influence access to and utilization of cancer screening services. As was discussed in last year's Annual Report to the Nation, the prevalence of mammography and cervical and colorectal cancer screening at recommended intervals also varies across the states. ${ }^{6}$

Marked variation among states was noted in the percentage of unstaged cases for all four cancers. These variations could be related to differences in medical practice, varying socioeconomic characteristics in the population, registry operations, or a combination of these factors. It is important to better understand the reasons for these differences because inadequate staging may result in suboptimal treatment.

\section{Limitations}

There are certain limitations in the data and methods that may influence the interpretations of the findings in this report. First, routinely collected statistics regarding cancer occurrence, as provided in this analysis, are commonly reported according to the major racial and ethnic populations-whites, blacks, APIs, AI/ANs, and Hispanics/Latinos. Such broad racial and ethnic groupings may mask wide variations in the cancer burden by country of origin, for example, 
among APIs (China, Japan, Philippines, Vietnam, etc.) ${ }^{126,127}$ and Hispanics/Latinos (Spain, Cuba, Puerto Rico, Mexico, etc. $)^{128,129}$ and by cultural characteristics that define other high-risk populations such as white residents in Appalachia, ${ }^{130}$ recent immigrants, blacks in the rural South, and over 560 American Indian tribes recognized by individual states and the federal government. ${ }^{12,131-133}$ Cancer rates for populations other than whites and blacks may be limited by problems in ascertaining race/ethnicity information from basic records (medical records, death certificates, and census reports). ${ }^{134,135}$ Furthermore, the assessment of long-term cancer trends is limited to white and black populations because annual population counts for other racial and ethnic populations are not available prior to 1990 .

Second, the survival data and most of the incidence data presented in this report are taken from the SEER registries. The SEER registries were selected to be a reasonably representative subset of the U.S. population, with a higher representation of racial and ethnic minorities. Therefore, cancer statistics generated from SEER areas may differ in some respects from cancer statistics throughout the country because SEER areas tend to have more foreign-born individuals and be more urban populations compared with non-SEER areas. $^{136}$

Third, we used two different statistical methods to describe cancer trends. A single linear model was used to describe short-term trends (1992-2001) in cancer incidence and death rates by race and ethnicity. In contrast, long-term patterns (1975-2001) for all races and ethnicities combined were characterized using the joinpoint method. Analyses of data by these two approaches may in some circumstances lead to different results. For example, the prostate cancer incidence rate decreased by $2.5 \%$ per year from 1992-2001, whereas in the joinpoint model it increased by $1.4 \%$ per year from 1995-2001, after an $11.4 \%$ per year decrease from 1992-1995. The joinpoint model is a more flexible and accurate approach to identify the years in which significant changes in trends occurred, but cannot always be employed for analyses of trends by race and ethnicity because of the limited time period with available data.

\section{Future Directions}

The long-term goals of basic and clinical research, cancer surveillance, and cancer control are activities to eliminate suffering and death from cancer. This will come about by striving to prevent the onset as well as the progression of cancer; identifying cancers at the earliest stage of disease; eliminating cancer through targeted treatments; and controlling cancers that can- not be eliminated so they become manageable, chronic diseases.

Considerable reduction in the suffering and death from cancer in the U.S. could be achieved by reaching all segments of the population with high-quality prevention, early detection, and treatment services. ${ }^{137}$ Disparities in access to and quality of care have been documented throughout the cancer spectrum. For example, the uninsured and those with only Medicare or Medicaid may lack access to effective smoking cessation therapies. ${ }^{137}$ Individuals with low incomes, those without health insurance, and those who immigrated to the U.S. within the last 10 years have a lower prevalence of mammography and colorectal and Papanicolaou smear testing than other population groups. ${ }^{30}$ Geographic variations also exist in the utilization of recommended screening tests ${ }^{6}$ and in the stage of disease at diagnosis for cancers that are detectable by screening (Table 7). In response to the documentation of healthcare disparities for racial and ethnic minorities and other medically underserved groups, federal programs targeted at reducing disparities have been created and strengthened. For example, the Quality of Cancer Care Committee (QCCC), organized by NCI in 2000, has sponsored several interagency projects to examine how the best available scientific evidence concerning intervention effectiveness can inform federal-level decision making regarding cancer care. In one QCCC project, three federal agencies (the NCI, the CDC, and the Health Resources and Services Administration) are collaborating with the nonprofit Institute for Healthcare Improvement to enhance screening and follow-up care for patients with breast, cervical, and colorectal cancers in community health centers for the medically underserved. ${ }^{139}$ The National Breast and Cervical Cancer Early Detection Program created by the CDC in 1991 has provided over 4 million screening examinations to underserved women. However, estimates are that this program reaches only $12-15 \%$ of eligible women nationally. ${ }^{140}$ In 2000 , the NIH established the National Center on Minority Health and Health Disparities to lead and coordinate $\mathrm{NIH}$ efforts to improve the health of minority and medically underserved people. In 2001, the Center to Reduce Cancer Health Disparities was created within the NCI to stimulate research to address cancer health disparities.

There also has been growing recognition of gaps in the delivery of high-quality cancer care in recent years. $^{31}$ In 1999, the National Cancer Policy Board issued a comprehensive report on the state of the cancer care system, which concluded that some individuals with cancer do not receive care known to be effective for their condition. ${ }^{138}$ Recommendations in- 
cluded the development and use of evidence-based guidelines for all aspects of cancer care; the development of a core set of quality measures that can be used to measure and monitor the quality of care; the enhancement of services for the uninsured and underinsured to ensure entry to, and equitable treatment within, the cancer care system; and research to understand why specific segments of the population do not receive appropriate care. Partnerships to nurture an integrated approach to fighting cancer and to ensure that research discoveries are translated into clinical and public health interventions that can be delivered to all who need them have been embraced by many private and public institutions, several of which have made commitments to programs and activities that are building blocks for this effort. ${ }^{139}$ For example, the NAACCR is actively involved in extending its standardization activities beyond the cancer registry community to foster and contribute to a national health information structure.

In addition to the better application of existing knowledge, leaders in the scientific community forecast an era of unprecedented progress in cancer research through applying knowledge of the molecular and cellular mechanisms that underlie the initiation and progression of cancer. Areas of particular interest, highlighted in the NCI's research priorities, include seven strategic priority areas: molecular epidemiology; integrated cancer biology; the strategic development of cancer interventions; prevention, early detection, and prediction; an integrated clinical trials system; overcoming health disparities; and bioinformatics. ${ }^{141}$ The development of imatinib mesylate for the treatment of CML is an example of a treatment breakthrough resulting from understanding the molecular abnormality involved in a particular type of cancer. ${ }^{100}$ In addition to treatment advances, a better understanding of the basis of cancer has led to more effective prevention strategies, the development of improved tests for early detection, more precise diagnostic methods, and more powerful treatment approaches. Transdisciplinary research efforts have the potential to enhance our understanding of the interaction of genetic and environmental risk factors for tobacco addiction and obesity, leading to the improved prevention and treatment of the major cancer risk factors in the U.S. population. However, for research advances to impact cancer incidence and death rates, the translation of research discoveries to the widespread and equitable delivery of preventive and clinical services must be expedited.

In the 21 st century, cancer surveillance will play a critical role in monitoring the nation's progress against cancer. The Annual Report to the Nation is just one facet of ongoing collaborations among the ACS, the CDC's NPCR and NCHS, the NCI's SEER Program, and the NAACCR. Through collaborative and coordinated initiatives and programs, these organizations are working to improve the comprehensiveness and quality of data used to focus cancer control efforts and track progress against cancer. In the future, the integrated and efficient exchange of standardized information among clinical, administrative, and public health data systems will enable better assessments of these successes in all populations and in all areas of the U.S.

\section{REFERENCES}

1. Wingo P, Ries L, Rosenberg H, Miller D, Edwards B. Cancer incidence and mortality, 1973-1995: a report card for the U.S. Cancer. 1998;82:1197-1207.

2. Wingo P, Ries L, Giovino G, et al. Annual report to the nation on the status of cancer, 1973-1996, with a special section on lung cancer and tobacco smoking. J Natl Cancer Inst. 1999;91:675-690.

3. Ries L, Wingo P, Miller D, et al. Annual report to the nation on the status of cancer, 1973-1997, with a special section on colorectal cancer. Cancer. 2000;88:2398-2424.

4. Howe $\mathrm{H}$, Wingo $\mathrm{P}$, Thun $\mathrm{M}$, et al. Annual report to the nation on the status of cancer, 1973 through 1998, featuring cancers with increasing trends. J Natl Cancer Inst. 2001; 93:824-842.

5. Edwards B, Howe H, Ries L, et al. Annual report to the nation on the status of cancer, 1973-1999, featuring implications of age and aging on U.S. cancer burden. Cancer. 2002;94:2766-2792.

6. Weir HK, Thun MJ, Hankey BF, et al. Annual report to the nation on the status of cancer, 1975-2000, featuring the uses of surveillance data for cancer prevention and control. J Natl Cancer Inst. 2003;95:1276-1299.

7. Howe HL, Edwards BK, Young JL, et al. A vision for cancer incidence surveillance in the United States. Cancer Causes Control. 2003;14:663-672.

8. Fritz A, Percy C, Jack A, et al. International classification of diseases of oncology. Geneva: World Health Organization, 2000.

9. Ries LAG, Eisner MP, Kosary CL, et al. SEER cancer statistics review, 1975-2001. Available from URL http://www. seer.cancer.gov/csr/1975_2001/ [accessed May 5, 2004].

10. Kramarova E, Stiller CA. The international classification of childhood cancer. Int J Cancer. 1996;68:759-765.

11. Shambaugh EM, Weiss MA, Axtell LM, Ryan RF, Platz CE. Summary staging guide for the cancer Surveillance, Epidemiology, and End Results (SEER) reporting program. Bethesda, MD: National Institutes of Health, 1983.

12. Young JL Jr., Roffers SD, Ries LAG, Fritz AG, Hurlbut AA, editors. SEER summary staging manual-2000: codes and coding instructions, Bethesda (MD): National Cancer Institute; 2001 NIH Pub. No. 01-4969.

13. National Center for Health Statistics. Vital statistics of the United States, 1950-1999, Volume II. Mortality, Parts A and B. Washington, DC: Public Health Service, 2001. 
14. U.S. Department of Health Education and Welfare and National Center for Health Statistics. Manual of the international statistical classification of diseases, injuries, and causes of death, adapted for use in the United States. 8th revision. Washington, DC: Public Health Service, 1967.

15. World Health Organization. Manual of the international statistical classification of diseases, injuries, and causes of death, based on the recommendations of the ninth revision conference. Geneva: World Health Organization, 1977.

16. World Health Organization. International statistical classification of diseases and related health problems. 10th revision. Geneva: World Health Organization, 1992.

17. Anderson RN, Minino AM, Hoyert DL, Rosenberg HM. Comparability of cause of death between ICD-9 and ICD-10: preliminary estimates. Natl Vital Stat Rep. 2001; 49:1-32.

18. Surveillance, Epidemiology, and End Results (SEER) Program. SEER 1975-2001. SEER*Stat Database: IncidenceSEER 9 Regs, Nov 2003 Sub (1973-2001) < 19 Age Groups. Bethesda, MD: National Cancer Institute, DCCPS, Surveillance Research Program, Cancer Statistics Branch, 2004.

19. Kim HJ, Fay MP, Feuer EJ, Midthune DN. Permutation tests for joinpoint regression with applications to cancer rates. Stat Med. 2000;19:335-351.

20. Clegg LX, Feuer EJ, Midthune DN, Fay MP, Hankey BF. Impact of reporting delay and reporting error on cancer incidence rates and trends. J Natl Cancer Inst. 2002;94: $1537-1545$.

21. Kaplan EL, Meier P. Non parametric estimation from incomplete observations. J Am Stat Assoc. 1958;53:457-481.

22. Clegg LX, Li FP, Hankey BF, Chu K, Edwards BK. Cancer survival among US whites and minorities: a SEER (Surveillance, Epidemiology, and End Results) program population-based study. Arch Intern Med. 2002;162:1985-1993.

23. Cox DR. Regression models and life tables (with discussion). J R Stat Soc B. 1972;34:187-220.

24. SAS Institute Inc. SAS/STAT user's guide. Version 6. Cary, NC: SAS Institute, Inc., 1989.

25. Jemal A, Ward E, Anderson RN, Thun MJ. Influence of rules from the Tenth Revision of the International Classification of Diseases on U.S. cancer mortality trends. J Natl Cancer Inst. 2003;95:1727-1728.

26. Black WC, Welch HG. Advances in diagnostic imaging and overestimations of disease prevalence and the benefits of therapy. N Engl J Med. 1993;328:1237-1243.

27. Patz EF Jr., Goodman PC, Bepler G. Screening for lung cancer. N Engl J Med. 2000;343:1627-1633.

28. Feinstein AR, Sosin DM, Wells CK. The Will Rogers phenomenon. Stage migration and new diagnostic techniques as a source of misleading statistics for survival in cancer. N Engl J Med. 1985;312:1604-1608.

29. Ward E, Jemal A, Cokkinides V, et al. Cancer disparities by race/ethnicity and socioeconomic status. CA Cancer J Clin. 2004;54:78-93.

30. Swan J, Breen N, Coates RJ, Rimer BK, Lee NC. Progress in cancer screening practices in the United States: results from the 2000 National Health Interview Survey. Cancer. 2003;97:1528-1540.

31. Smedley B, Stith AY, Nelson AR. Unequal treatment: confronting racial and ethnic disparities in health care. Washington, DC: The National Academies Press, 2003:764.

32. Bach PB, Schrag D, Brawley OW, Galaznik A, Yakren S, Begg
CB. Survival of blacks and whites after a cancer diagnosis. JAMA. 2002;287:2106-2113.

33. Shavers VL, Brown ML. Racial and ethnic disparities in the receipt of cancer treatment. J Natl Cancer Inst. 2002;94: 334-357.

34. Legler JM, Feuer EJ, Potosky AL, Merrill RM, Kramer BS. The role of prostate-specific antigen (PSA) testing patterns in the recent prostate cancer incidence decline in the United States. Cancer Causes Control. 1998;9:519-527.

35. Hsing AW, Devesa SS. Trends and patterns of prostate cancer: what do they suggest? Epidemiol Rev. 2001;23:313.

36. Alavanja MC, Samanic C, Dosemeci M, et al. Use of agricultural pesticides and prostate cancer risk in the Agricultural Health Study cohort. Am J Epidemiol. 2003;157:800814.

37. Hankey BF, Feuer EJ, Clegg LX, et al. Cancer surveillance series: interpreting trends in prostate cancer-part I: evidence of the effects of screening in recent prostate cancer incidence, mortality, and survival rates. J Natl Cancer Inst. 1999;91:1017-1024.

38. Guileyardo JM, Johnson WD, Welsh RA, Akazaki K, Correa P. Prevalence of latent prostate carcinoma in two U.S. populations. J Natl Cancer Inst. 1980;65:311-316.

39. Breslow N, Chan CW, Dhom G, et al. Latent carcinoma of prostate at autopsy in seven areas. The International Agency for Research on Cancer, Lyons (France). Int J Cancer. 1977;20:680-688.

40. Etzioni R, Penson DF, Legler JM, et al. Overdiagnosis due to prostate-specific antigen screening: lessons from U.S. prostate cancer incidence trends. J Natl Cancer Inst. 2002;94: 981-990.

41. Cooperberg MR, Grossfeld GD, Lubeck DP, Carroll PR. National practice patterns and time trends in androgen ablation for localized prostate cancer. J Natl Cancer Inst. 2003;95:981-989.

42. Meng MV, Grossfeld GD, Sadetsky N, Mehta SS, Lubeck DP Carroll PR. Contemporary patterns of androgen deprivation therapy use for newly diagnosed prostate cancer. Urology. 2002;60:7-11; discussion, 11-12.

43. Smith M, Peto R. Prostate cancer deaths dropping in Europe, US, due to hormone therapy. The European Cancer Conference, Copenhagen (Denmark). Oncology Times XXV, 2003 October 25; page 24

44. Kupelian PA, Buchsbaum JC, Elshaikh MA, Reddy CA, Klein EA. Improvement in relapse-free survival throughout the PSA era in patients with localized prostate cancer treated with definitive radiotherapy: year of treatment an independent predictor of outcome. Int J Radiat Oncol Biol Phys. 2003;57:629-634.

45. Bennett CL, Ferreira MR, Davis TC, et al. Relation between literacy, race, and stage of presentation among low-income patients with prostate cancer. J Clin Oncol. 1998;16:31013104.

46. Harlan L, Brawley O, Pommerenke F, Wali P, Kramer B. Geographic, age, and racial variation in the treatment of local/regional carcinoma of the prostate. J Clin Oncol. 1995;13:93-100.

47. Burns DM, Lee L, Shen LZ, et al. Cigarette smoking in the United States. In: Burns DM, Garfinkel L, Samet JM, editors. Changes in cigarette-related disease risks and their implication for prevention and control. Monograph 8. Volume 2. Bethesda, MD: National Cancer Institute, 1997:13112. 
48. Orzechowski W, Walker R. The tax burden on tobacco: historical compilation 2001: impact and opportunity. Arlington, VA: Orzechowski and Walker, 2002.

49. Spira A, Ettinger DS. Multidisciplinary management of lung cancer. N Engl J Med. 2004;350:379-392.

50. Arriagada R, Bergman B, Dunant A, Le Chevalier T, Pignon JP, Vansteenkiste J. Cisplatin-based adjuvant chemotherapy in patients with completely resected non-small-cell lung cancer. $N$ Engl J Med. 2004;350:351-360.

51. Bach PB, Cramer LD, Warren JL, Begg CB. Racial differences in the treatment of early-stage lung cancer. $N$ Engl J Med. 1999;341:1198-1205.

52. Earle CC, Neumann PJ, Gelber RD, Weinstein MC, Weeks JC. Impact of referral patterns on the use of chemotherapy for lung cancer. J Clin Oncol. 2002;20:1786-1792.

53. Rossouw JE, Anderson GL, Prentice RL, et al. Risks and benefits of estrogen plus progestin in healthy postmenopausal women: principal results from the Women's Health Initiative randomized controlled trial. JAMA. 2002;288:321333.

54. Thun MJ, Namboodiri MM, Calle EE, Flanders WD, Heath CW Jr. Aspirin use and risk of fatal cancer. Cancer Res. 1993;53:1322-1327.

55. Chlebowski RT, Wactawski-Wende J, Ritenbaugh C, et al. Estrogen plus progestin and colorectal cancer in postmenopausal women. $N$ Engl J Med. 2004;350:991-1004.

56. Schottenfeld D, Winawer SJ. Cancer of the large intestine. In: Schottenfeld D, Fraumeni JF Jr., editors. Cancer epidemiology and prevention. New York: Oxford University Press, 1996:813-840.

57. Moertel CG, Fleming TR, Macdonald JS, et al. Levamisole and fluorouracil for adjuvant therapy of resected colon carcinoma. N Engl J Med. 1990;322:352-358.

58. Laurie JA, Moertel CG, Fleming TR, et al. Surgical adjuvant therapy of large-bowel carcinoma: an evaluation of levamisole and the combination of levamisole and fluorouracil. The North Central Cancer Treatment Group and the Mayo Clinic. J Clin Oncol. 1989;7:1447-1456.

59. Schrag D, Cramer LD, Bach PB, Begg CB. Age and adjuvant chemotherapy use after surgery for stage III colon cancer. J Natl Cancer Inst. 2001;93:850-857.

60. Hodgson DC, Fuchs CS, Ayanian JZ. Impact of patient and provider characteristics on the treatment and outcomes of colorectal cancer. J Natl Cancer Inst. 2001;93:501-515.

61. Potosky AL, Harlan LC, Kaplan RS, Johnson KA, Lynch CF. Age, sex, and racial differences in the use of standard adjuvant therapy for colorectal cancer. J Clin Oncol. 2002; 20:1192-1202.

62. Schrag D, Gelfand SE, Bach PB, Guillem J, Minsky BD, Begg CB. Who gets adjuvant treatment for stage II and III rectal cancer? Insight from Surveillance, Epidemiology, and End Results-Medicare. J Clin Oncol. 2001;19:3712-3718.

63. Cooper GS, Yuan Z, Landefeld CS, Rimm AA. Surgery for colorectal cancer: race-related differences in rates and survival among Medicare beneficiaries. Am J Public Health. 1996;86:582-586.

64. Ghafoor A, Jemal A, Ward E, Cokkinides V, Smith R, Thun MJ. Trends in breast cancer by race and ethnicity. $C A$ Cancer J Clin. 2003;53:342-355.

65. Harlan LC, Abrams J, Warren JL, Clegg L, Stevens J, BallardBarbash R. Adjuvant therapy for breast cancer: practice patterns of community physicians. J Clin Oncol. 2002;20: 1809-1817.

66. Mariotto A, Feuer EJ, Harlan LC, Wun LM, Johnson KA,
Abrams J. Trends in use of adjuvant multi-agent chemotherapy and tamoxifen for breast cancer in the United States: 1975-1999. J Natl Cancer Inst. 2002;94:1626-1634.

67. American Cancer Society. Cancer facts \& figures 2004. Atlanta: American Cancer Society, Inc., 2004.

68. Griggs JJ, Sorbero ME, Stark AT, Heininger SE, Dick AW. Racial disparity in the dose and dose intensity of breast cancer adjuvant chemotherapy. Breast Cancer Res Treat. 2003;81:21-31.

69. Mandelblatt JS, Kerner JF, Hadley J, et al. Variations in breast carcinoma treatment in older Medicare beneficiaries: is it black or white. Cancer. 2002;95:1401-1414.

70. Newman LA, Kuerer HM, Harper T, et al. Special considerations in breast cancer risk and survival. J Surg Oncol. 1999;71:250-260.

71. Bradley CJ, Given CW, Roberts C. Disparities in cancer diagnosis and survival. Cancer. 2001;91:178-188.

72. Chu KC, Lamar CA, Freeman HP. Racial disparities in breast carcinoma survival rates: separating factors that affect diagnosis from factors that affect treatment. Cancer. 2003;97:2853-2860.

73. Eley JW, Hill HA, Chen VW, et al. Racial differences in survival from breast cancer. Results of the National Cancer Institute Black/White Cancer Survival Study. JAMA. 1994; 272:947-954

74. Chen VW, Correa P, Kurman RJ, et al. Histological characteristics of breast carcinoma in blacks and whites. Cancer Epidemiol Biomarkers Prev. 1994;3:127-135.

75. Elledge RM, Clark GM, Chamness GC, Osborne CK. Tumor biologic factors and breast cancer prognosis among white, Hispanic, and black women in the United States. J Natl Cancer Inst. 1994;86:705-712.

76. Hershman D, Weinberg M, Rosner Z, et al. Ethnic neutropenia and treatment delay in African American women undergoing chemotherapy for early-stage breast cancer. J Natl Cancer Inst. 2003;95:1545-1548.

77. Eltom MA, Jemal A, Mbulaiteye SM, Devesa SS, Biggar RJ. Trends in Kaposi's sarcoma and non-Hodgkin's lymphoma incidence in the United States from 1973 through 1998. J Natl Cancer Inst. 2002;94:1204-1210.

78. Freedman AS, Nadler LM. Non-Hodgkin's lymphomas. In: Holland JF, Frei E, editors. Cancer medicine 6. Volume 2. Hamilton, Ontario: BC Decker Inc., 2003:2189-2210.

79. Harris NL, Jaffe ES, Stein H, et al. A revised EuropeanAmerican classification of lymphoid neoplasms: a proposal from the International Lymphoma Study Group. Blood. 1994;84:1361-1392.

80. Armitage JO, Weisenburger DD. New approach to classifying non-Hodgkin's lymphomas: clinical features of the major histologic subtypes. Non-Hodgkin's Lymphoma Classification Project. J Clin Oncol. 1998;16:2780-2795.

81. Chen MG, Prosnitz LR, Gonzalez-Serva A, Fischer DB. Results of radiotherapy in control of stage I and II nonHodgkin's lymphoma. Cancer. 1979;43:1245-1254.

82. McLaughlin P, Fuller LM, Velasquez WS, Sullivan-Halley JA, Butler JJ, Cabanillas F. Stage I-II follicular lymphoma. Treatment results for 76 patients. Cancer. 1986;58:15961602.

83. Ezdinli EZ, Harrington DP, Kucuk O, Silverstein MW, Anderson J, O'Connell MJ. The effect of intensive intermittent maintenance therapy in advanced low-grade nonHodgkin's lymphoma. Cancer. 1987;60:156-160. 
84. Jones SE, Grozea PN, Miller TP, et al. Chemotherapy with cyclophosphamide, doxorubicin, vincristine, and prednisone alone or with levamisole or with levamisole plus BCG for malignant lymphoma: a Southwest Oncology Group Study. J Clin Oncol. 1985;3:1318--324.

85. Young RC, Johnson RE, Canellos GP, et al. Advanced lymphocytic lymphoma: randomized comparisons of chemotherapy and radiotherapy, alone or in combination. Cancer Treat Rep. 1977;61:1153-1159.

86. DeVita VT Jr., Canellos GP, Chabner B, Schein P, Hubbard SP, Young RC. Advanced diffuse histiocytic lymphoma, a potentially curable disease. Lancet. 1975;1:248-250.

87. Coltman C. CHOP is curative in thirty percent of patients with large cell lymphoma: a twelve year Southwest Oncology Group follow-up. Volume 71. New York: Park Row, 1986.

88. Blum KA, Bartlett NL. Antibodies for the treatment of diffuse large cell lymphoma. Semin Oncol. 2003;30:448-456.

89. Philip T, Guglielmi C, Chauvin F. Autologous bone marrow transplantation versus conventional chemotherapy (DHAP) in relapsed non-Hodgkin's lymphoma: final analysis of the PARMA randomized study [abstract]. Proc Am Soc Clin Oncol. 1995;14:390.

90. Blay J, Gomez F, Sebban C, et al. The International Prognostic Index correlates to survival in patients with aggressive lymphoma in relapse: analysis of the PARMA trial. Parma Group. Blood. 1998;92:3562-3568.

91. Jemal A, Devesa SS, Hartge P, Tucker MA. Recent trends in cutaneous melanoma incidence among whites in the United States. J Natl Cancer Inst. 2001;93:678-683.

92. Armstrong BK, English DR. Cutaneous malignant melanoma. In: Schottenfeld D, Fraumeni JF Jr., editors. Cancer epidemiology and prevention. New York: Oxford University Press, 1996:1521.

93. Hall HI, Miller DR, Rogers JD, Bewerse B. Update on the incidence and mortality from melanoma in the United States. J Am Acad Dermatol. 1999;40:35-42.

94. Morton DL. Current management of malignant melanoma. Ann Surg. 1990;212:123-124.

95. Morton DL, Foshag LJ, Hoon DS, et al. Prolongation of survival in metastatic melanoma after active specific immunotherapy with a new polyvalent melanoma vaccine. Ann Surg. 1992;216:463-482.

96. Agarwala S. Improving survival in patients with high-risk and metastatic melanoma: immunotherapy leads the way. Am J Clin Dermatol. 2003;4:333-346.

97. Garcia-Manero GKH, Schiffer CA. Adult acute lymphocytic leukemia. In: Holland JF, Frei E, editors. Cancer medicine 6. Volume 2. Hamilton, Ontario: BC Decker Inc., 2003: $2125-2134$.

98. Gottlieb AJ, Weinberg V, Ellison RR, et al. Efficacy of daunorubicin in the therapy of adult acute lymphocytic leukemia: a prospective randomized trial by cancer and leukemia group B. Blood. 1984;64:267-274.

99. Schiffer CA. Acute myeloid leukemia in adults. In: Holland JF, Frei E, editors. Cancer medicine 6. Volume 2. Hamilton, Ontario: BC Decker Inc., 2003:2095-2116.

100. Silver RT. Chronic myeloid leukemia. In: Holland JF, Frei E, editors. Cancer medicine 6. Volume 2. Hamilton, Ontario: BC Decker Inc., 2003:2117-2124.

101. Druker BJ, Talpaz M, Resta DJ, et al. Efficacy and safety of a specific inhibitor of the BCR-ABL tyrosine kinase in chronic myeloid leukemia. N Engl J Med 2001;344:10311037.
102. Kantarjian H, Sawyers C, Hochhaus A, et al. Hematologic and cytogenetic responses to imatinib mesylate in chronic myelogenous leukemia. N Engl J Med. 2002;346:645-652.

103. Kadan-Lottick NS, Ness KK, Bhatia S, Gurney JG. Survival variability by race and ethnicity in childhood acute lymphoblastic leukemia. JAMA. 2003;290:2008-2014.

104. Carroll WL. Race and outcome in childhood acute lymphoblastic leukemia. JAMA. 2003;290:2061-2063.

105. Pui CH, Sandlund JT, Pei D, et al. Results of therapy for acute lymphoblastic leukemia in black and white children. JAMA. 2003;290:2001-2007.

106. Chow WH, Devesa SS, Warren JL, Fraumeni JF Jr. Rising incidence of renal cell cancer in the United States. JAMA. 1999;281:1628-1631.

107. Jayson M, Sanders H. Increased incidence of serendipitously discovered renal cell carcinoma. Urology. 1998;51: 203-205.

108. Leslie JA, Prihoda T, Thompson IM. Serendipitous renal cell carcinoma in the post-CT era: continued evidence in improved outcomes. Urol Oncol. 2003;21:39-44.

109. Flegal KM, Carroll MD, Kuczmarski RJ, Johnson CL. Overweight and obesity in the United States: prevalence and trends, 1960-1994. Int J Obes Relat Metab Disord. 1998;22: $39-47$.

110. Flegal KM, Carroll MD, Ogden CL, Johnson CL. Prevalence and trends in obesity among US adults, 1999-2000. JAMA. 2002;288:1723-1727.

111. Chow WH, Gridley G, Fraumeni JF Jr., Jarvholm B. Obesity, hypertension, and the risk of kidney cancer in men. $N$ Engl J Med. 2000;343:1305-1311.

112. Espey DK, Paisano RE, Cobb N. Cancer mortality among American Indians and Alaska Natives: regional differences, 1994-1998. Rockville, MD: Indian Health Service, 2003.

113. Richie JP, Kantoff PW. Renal cell carcinoma. In: Holland JF, Frei E, editors. Cancer medicine 6. Volume 2. Hamilton, Ontario: BC Decker Inc., 2003:1675-1682.

114. Balis F, Holcenberg J, Blaney S. General principles of chemotherapy. In: Pizzo P, Poplack D, editors. Principles and practice of pediatric oncology. Philadelphia: Lippincott Williams \& Wilkins, 2002:237-308.

115. Liu L, Krailo M, Reaman GH, Bernstein L. Childhood cancer patients' access to cooperative group cancer programs: a population-based study. Cancer. 2003;97:1339-1345.

116. Reaman G. Pediatric oncology: principles and practice. In: Holland JF, Frei E, editors. Cancer medicine 6. Volume 2. Hamilton, Ontario: BC Decker Inc., 2003:2291-2293.

117. Bleyer WA, Tejeda H, Murphy SB, et al. National cancer clinical trials: children have equal access; adolescents do not. J Adolesc Health. 1997;21:366-373.

118. Mandelblatt J, Andrews H, Kao R, Wallace R, Kerner J. The late-stage diagnosis of colorectal cancer: demographic and socioeconomic factors. Am J Public Health. 1996;86:17941797.

119. Richardson JL, Langholz B, Bernstein L, Burciaga C, Danley $K$, Ross RK. Stage and delay in breast cancer diagnosis by race, socioeconomic status, age and year. Br J Cancer. 1992;65:922-926.

120. Wells BL, Horm JW. Stage at diagnosis in breast cancer: race and socioeconomic factors. Am J Public Health. 1992; 82:1383-1385.

121. Breen N, Figueroa JB. Stage of breast and cervical cancer diagnosis in disadvantaged neighborhoods: a prevention policy perspective. Am J Prev Med. 1996;12:319-326. 
122. Campbell RJ, Ferrante JM, Gonzalez EC, Roetzheim RG, Pal N, Herold A. Predictors of advanced stage colorectal cancer diagnosis: results of a population-based study. Cancer Detect Prev. 2001;25:430-438.

123. Kaffashian F, Godward S, Davies T, Solomon L, McCann J, Duffy SW. Socioeconomic effects on breast cancer survival: proportion attributable to stage and morphology. $\mathrm{Br} J \mathrm{Can}$ cer. 2003;89:1693-1696.

124. Riley GF, Potosky AL, Lubitz JD, Brown ML. Stage of cancer at diagnosis for Medicare HMO and fee-for-service enrollees. Am J Public Health. 1994;84:1598-1604.

125. Ayanian JZ, Kohler BA, Abe T, Epstein AM. The relation between health insurance coverage and clinical outcomes among women with breast cancer. N Engl J Med. 1993;329: 326-331.

126. Miller BA, Kolonel LN, Bernstein L, et al. Racial/ethnic patterns of cancer in the United States 1988-1992. Bethesda, MD: National Cancer Institute, 1996.

127. Deapen D, Liu L, Perkins C, Bernstein L, Ross RK. Rapidly rising breast cancer incidence rates among Asian-American women. Int J Cancer. 2002;99:747-750.

128. Trapido EJ, Burciaga Valdez R, Obeso JL, Strickman-Stein N, Rotger A, Perez-Stable EJ. Epidemiology of cancer among Hispanics in the United States. J Natl Cancer Inst Monograph 1995;18:17-28.

129. Wolfgang PE, Semeiks PA, Burnett WS. Cancer incidence in New York City Hispanics, 1982 to 1985. Ethn Dis. 1991;1: 263-272.

130. Friedell GH, Ross FE, Tucker TC, et al. Cervical cancer in Kentucky. J Ky Med Assoc. 2000;98:398-405.

131. Bureau of Indian Affairs. Available from URL: http://www. doi.gov/bureau-indian-affairs.html [accessed on February 27, 2004].

132. Haynes MA, Smedley BD. The unequal burden of cancer: an assessment of NIH research and programs for ethnic minorities and the medically underserved. Washington, DC: National Academy Press, 1999.
133. Sugarman JR, Holliday M, Ross A, Castorina J, Hui Y. Improving American Indian cancer data in the Washington State Cancer Registry using linkages with the Indian Health Service and tribal records. Cancer. 1996;78:1564-1568.

134. Poe GS, Powell-Griner E, McLaughlin JK, Placek PJ, Thompson GB, Robinson K. Comparability of the death certificate and the 1986 National Mortality Followback Survey. Vital Health Stat 2. 1993;(118):1-53.

135. Rosenberg HM, Maurer JD, Sorlie PD, et al. Quality of death rates by race and Hispanic origin: a summary of current research, 1999. Vital Health Stat 2. 1999;(128):1-13.

136. Characteristics of the SEER population compared with the total United States population. Available from URL: http:// www.seer.cancer.gov/registries/characteristics.html [accessed February 27, 2004]

137. Curry SJ, Byers T, Hewitt M. Fulfilling the potential of cancer prevention and early detection. Washington, DC: The National Academies Press, 2003.

138. Institute of Medicine National Research Council. Ensuring quality cancer care. In: Hewitt M, Simone JV, editors. National Cancer Policy Board, Institute of Medicine and Commission on Life Sciences, National Research Council. Washington, DC: National Academy Press, 1999:246.

139. Lipscomb J, Donaldson MS. Outcomes research at the National Cancer Institute: measuring, understanding, and improving the outcomes of cancer care. Clin Ther. 2003;25: 699-712.

140. Centers for Disease Control and Prevention. Implementing recommendations for the early detection of breast and cervical cancer among low income women. MMWR Morb Mortal Wkly Rep. 2000;49:37-50.

141. U.S. Department of Health and Human Services. The Nation's investment in cancer research. A plan and budget proposal for Fiscal Year 2005. Bethesda, MD: National Cancer Institute, 2003. 